\title{
Experimental identification of the lateral human-structure interaction mechanism and assessment of the inverted-pendulum biomechanical model.
}

\author{
S.P.Carroll ${ }^{\mathrm{a}, *}$, J.S.Owen ${ }^{\mathrm{a}}$, M.F.M.Hussein ${ }^{\mathrm{b}}$ \\ ${ }^{a}$ Centre for Structural Engineering and Construction (CSEC), \\ University of Nottingham, University Park, Nottingham, NG7 2RD, United Kingdom \\ ${ }^{b}$ Institute of Sound and Vibration Research, \\ University of Southampton, Highfield Campus, Southampton, SO17 1BJ, United Kingdom
}

\begin{abstract}
Within the context of crowd-induced lateral bridge vibration, human-structure interaction (HSI) is a widely studied phenomenon. Central to this study is the self-excited component of the ground reaction force (GRF). This force harmonic, induced by a walking pedestrian, resonates with lateral deck motion, irrespective of the pedestrian's pacing frequency. Its presence can lead to positive feedback between pedestrian GRFs and structural motion. Characterisation of the self-excited force as equivalent structural mass and damping has greatly improved the understanding HSI and its role in developing lateral dynamic instability. However, despite this evolving understanding, a key question has remained unanswered; what are the features of a pedestrian's balance response to base motion that give rise to the self-excited force? The majority of the literature has focused on the effects of HSI with the underlying mechanism receiving comparatively little attention. This paper presents data from experimental testing in which 10 subjects walked individually on a laterally oscillating treadmill. Lateral deck motion as well as the GRFs imposed by the subject were recorded. Three-dimensional motion capture equipment was used to track the position of visual markers mounted on the subject. Thus whole body response to base motion was captured in addition to the GRFs generated. The data presented herein supports the authors' previous findings that the self-excited force is a frequency sideband harmonic resulting from amplitude modulation of the lateral GRF. The gait behaviour responsible for this amplitude modulation is a periodic modulation of stride width in response to a sinusoidally varying inertia force induced by deck motion. In a separate analysis the validity of the passive inverted pendulum model, stabilised by active control of support placement was confirmed. This was established through comparison of simulated and observed frontal plane CoM motion. Despite the relative simplicity of this biomechanical model, remarkable agreement was observed.
\end{abstract}

Keywords: human-induced vibration, biomechanics, motion capture, footbridge, amplitude modulation, lateral dynamic instability, inverted pendulum

\section{Introduction}

Field observations of bridges exhibiting large amplitude lateral oscillations under the influence of pedestrian traffic have grown in number $[1,2,3,4,5]$ since the publication of Dallard et al's discussion of London's Millennium Bridge [6]. A central theme linking these loading events is that the response amplitudes observed are uncharacteristically large considering the number of pedestrians present on the structure. Thus the logical conclusion is that some form of positive feedback is occurring between the walking crowd and the oscillating structure. The unknown nature of this interaction continues to motivate research efforts.

\footnotetext{
*Corresponding author. Tel: +44 (0)115 8468933

Email address: sean.carroll@nottingham.ac.uk (S.P.Carroll)
} 
The initial assumption of an interaction mechanism based on step synchronisation between pedestrians and an oscillating bridge deck is now thought to be preceded by a more subtle interaction $[4,5]$. This humanstructure interaction (HSI) (as opposed to human-structure synchronisation) results in a force harmonic within the ground reaction force (GRF) spectrum, that resonate with bridge deck motion [7]. These force harmonics are referred to as self-excited forces due to their dependence on and resonance with, deck motion.

Based on an extensive experimental campaign, Ingölfsson et al. [8] have further characterised the selfexcited force as a mass and damping influence on the oscillating structure. Despite these advances, a fundamental question has remained unanswered; what is the lateral HSI mechanism? Put another way, what is it about a pedestrian's balance response to base motion that gives rise to the self-excited force? The GRF (of which the self-excited force is a component) is a direct result of body mass accelerations, which are themselves determined by the pedestrian's response to deck motion. As such, to further understand HSI, one must first consider pedestrian stability and balance behaviour while walking in the presence of lateral deck motion.

An experimental campaign was therefore designed in which test subjects walked on a laterally oscillating treadmill. A range of tests were carried out, with varying oscillation frequencies and amplitudes imposed on the deck. GRFs were directly measured using deck-mounted load cells. In addition, each subject was instrumented with 31 active visual markers in order to record 3-dimensional whole body motion. In broad terms, the objective of this campaign is to not only capture the GRFs generated while walking on an oscillating deck, but also to identify the associated balance behaviour responsible for their generation.

Preliminary results relating to two participants in this campaign have been previously reported by the authors [9]. That preliminary data set identifies foot placement position as being central to the development of the self-excited force. Amplitude modulation (AM) was identified as the mechanism through which sideband force harmonics are generated in the GRF spectrum. One of the sidebands was identified as the self-exited force harmonic. The data and discussion presented herein further support these preliminary findings.

Understanding the HSI mechanism is itself an important milestone, however developing robust interaction models based on this understanding offers the ability to predict future bridge response. Attempts to model lateral HSI have been greatly advanced by Macdonald's investigation of the inverted pendulum biomechanical model [10]. This single degree of freedom model, actively stabilised on a step-by-step basis [11, 12, 13], through control of pendulum support position, allows HSI to be directly simulated. Macdonald's modelling approach has been adopted by others, including the authors of this work, for the simulation of multipedestrian HSI $[14,15,16]$. However, there remains a question regarding the validity of the centre of mass (CoM) motion simulated by the inverted pendulum model on an oscillating structure. The source of this uncertainty will be elaborated on below.

The motion capture data obtained in this campaign allows the trajectory of the pedestrian's CoM to be estimated and tracked. This provides an excellent opportunity to compare observed CoM motion with that predicted by the inverted pendulum model. Thus the uncertainty regarding inverted pendulum model validity will also be addressed in this paper.

In section 2 details of the experimental test campaign are provided including a brief description of the motion capture equipment, marker placement and the oscillating treadmill test rig. Data acquisition and processing is discussed in section 3. Section 4 contains an analysis of experimentally observed gait behaviour and further supports the authors previous analysis [9], linking gait behaviour to the self-excited force. This is followed by an assessment of the validity of the inverted pendulum biomechanical model for use on an oscillating deck. The assessment is facilitated by the direct comparison of experimentally observed and simulated CoM motion. Finally, section 6 summarised the main conclusions.

\section{Experimental investigation}

The experimental campaign required test subjects to walk (individually) on a laterally oscillating treadmill. During each test, the lateral component of the GRF was measured using 4 deck mounted Zemic bending beam load cells (490 N capacity each). Lateral deck displacement and acceleration were also recorded via 
a linear variable differential transformer (LVDT) and Schaevitz linear servo accelerometer. The position of 31 visual markers, mounted on the test subject was simultaneously recorded using a Coda mpx30 generalpurpose 3D motion tracking system.

All testing was carried out at the University of Nottingham's Human Performance Laboratory between March and May of 2012. The test protocol received ethical approval from the Faculty (of Engineering) Research Ethics Committee within the University of Nottingham. Ten subjects took part in the test campaign. The age, height and weight of all participants is shown in table 1 . The majority of anthropometric data in the literature has been obtained from male subjects. To ensure congruency and in order to utilise this data as effectively as possible, only male subjects were recruited for this campaign. This is justifiable when one considers that there is no reason to suspect that male subjects will behave (statistically) significantly differently to female subjects. It is therefore assumed, until proven otherwise, that the behaviours observed and conclusions drawn are equally applicable to both sexes.

\begin{tabular}{llll}
\multicolumn{4}{c}{ Table 1: Test subject data. } \\
\hline Subject & Height $(\mathrm{m})$ & Mass $(\mathrm{kg})$ & Age (Years) \\
\hline 1 & 1.73 & 82.05 & 28 \\
2 & 1.82 & 96.20 & 30 \\
3 & 1.74 & 63.05 & 27 \\
4 & 1.84 & 98.95 & 28 \\
5 & 1.68 & 85.45 & 34 \\
6 & 1.88 & 90.95 & 21 \\
7 & 1.82 & 87.20 & 26 \\
8 & 1.61 & 75.00 & 31 \\
9 & 1.80 & 74.05 & 28 \\
10 & 1.76 & 82.70 & 34 \\
\hline Mean & 1.77 & 83.11 & 28.7 \\
\hline
\end{tabular}

The test protocol for an individual subject was as follows;

- The subject was familiarised with the oscillating treadmill and all safety procedures.

- The subject was instrumented with gait analysis wands and visual markers.

- The subject had a further unrecorded period of familiarisation walking on the treadmill without lateral motion, lasting approximately 10 minutes. During this time a comfortable walking speed was selected by the subject.

- The subject was then recorded walking without lateral motion imposed on the deck. This data served as a baseline for the subject's walking behaviour.

- After baseline tests were completed, the subject was recorded walking while lateral oscillations were imposed on the deck, referred to hereafter as dynamic tests. For all dynamic tests, the subject walked at a speed that was self selected for each test. This speed was maintained throughout each 20 second test.

- Rest breaks were imposed every 2-3 minutes during the course of the test session.

Five oscillation amplitudes were tested, $5 \mathrm{~mm}, 10 \mathrm{~mm}, 20 \mathrm{~mm}, 35 \mathrm{~mm}$ and $50 \mathrm{~mm}$. Within each amplitude, 9 oscillation frequencies were tested ranging from $0.3 \mathrm{~Hz}$ to $1.1 \mathrm{~Hz}$ in $0.1 \mathrm{~Hz}$ increments, resulting in a 45 point test matrix. 


\subsection{Oscillating treadmill test rig}

The design of the oscillating treadmill test rig, Fig. 1 (a), hereafter referred to as 'the rig', and validation of GRF data obtained from the rig, have been reported in [9]. Therefore a concise functional description of the apparatus is provided herein. The rig consists of three frames, Fig. 1 (d). The base frame is the lowermost frame and remains stationary. The chassis frame rests on 4 carriages that travel along linear guide rails fixed to the base frame. The chassis frame is driven laterally in a sinusoidal reciprocating motion by a drive arm connected to the motor, see Fig. 1 (b). The uppermost treadmill frame is suspended from the chassis frame by 4 hangers, Fig. 1 (c). The self-weight of the treadmill frame and any vertical imposed loads are resisted by the hangers. The treadmill frame is restrained laterally by 4 bending beam load cells, the means through which the lateral GRF is recorded. The treadmill belt is driven by a second motor (not shown) mounted on the chassis frame. The deck walking area measures $1 \mathrm{~m}$ wide $\times 1.5 \mathrm{~m}$ long. A horizontal bar/handle was placed across the front of the treadmill to provide extra stability should a test subject feel particularly unstable. Subjects were instructed not to touch the bar unless absolutely necessary, after which the test data was discounted.

\subsection{Motion capture system}

A Coda 3D motion tracking system was used [17], consisting of 2 measurement units, each containing 3 pre-aligned cameras, wall mounted on either side of the test rig. The cameras track the position of active markers (infra-red LEDs) in the measurement volume, achieving a position resolution of $0.1 \mathrm{~mm}$ horizontally and vertically and a distance resolution of $0.3 \mathrm{~mm}$. Markers were placed directly over bony landmarks and attached to gait analysis wands strapped to the body, Fig. 2. More specifically, markers were positioned on the lateral aspect of the heel and at the end of the $5^{\text {th }}$ metatarsal, at the lateral malleolus (ankle) and on the lateral aspect of the medio-lateral knee axis. Tibial and femoral wands, containing two additional markers each, were strapped to the subject's lower leg and thigh. A pelvic frame containing 6 markers was attached to the subject at waist level.

Four markers were placed on the upper torso; one on each acromion (shoulder), one on the back of the neck over the $\mathrm{C} 7$ vertebra and one over the jugular notch. A marker was also placed above the ear, at eye level. Finally, a marker was placed on the lateral aspect of each elbow and wrist in order to capture arm motion.

During testing subjects wore a sleeveless shirt and shorts, Fig. 2 (b) \& (c). Subjects were not permitted to wear any form of footwear. This may at first appear counter intuitive as pedestrians walking on a bridge rarely walk barefoot. However, the emphasis in this investigation is on understanding biomechanical response in the face of lateral deck motion. The somatosensory information received through the sole of the foot is undoubtedly altered if a person wears shoes. Whether or not this information is altered to the extent that it has an influence on the actual balance behaviour is less certain. It was therefore decided to remove any potential variability introduced by footwear.

\section{Data acquisition and processing}

A sampling rate of $250 \mathrm{~Hz}$ was selected for all treadmill-borne data to provide good time domain resolution. A National Instruments SCXI-1000 chassis, housing a SCXI-1100, 32 channel multiplexer amplifier (receiving LVDT and accelerometer signals) and SCXI-1520 strain bridge (receiving load cell signals) was used to coordinate data logging and signal conditioning. The chassis was also used to trigger simultaneous logging of the marker data. All marker data was sampled at $100 \mathrm{~Hz}$ using a CODA ActiveHub computer from Charnwood Dynamics Ltd.

Considering the sampling frequency of $250 \mathrm{~Hz}$, all analogue signals were filtered at $100 \mathrm{~Hz}$ to avoid aliasing. The accelerometer and LVDT recording deck motion were further digitally low pass filtered with 4th order Butterworth (BW4) filters. A cutoff frequency of $2 \mathrm{~Hz}$ was selected for deck displacement as deck oscillations do not exceed $1.1 \mathrm{~Hz}$. Because deck acceleration data is used in the extraction of GRFs, a higher cutoff frequency of $5 \mathrm{~Hz}$ was selected to allow higher frequency force harmonics to be obtained. The load cells were digitally low pass filtered at $6 \mathrm{~Hz}$ (BW4). 

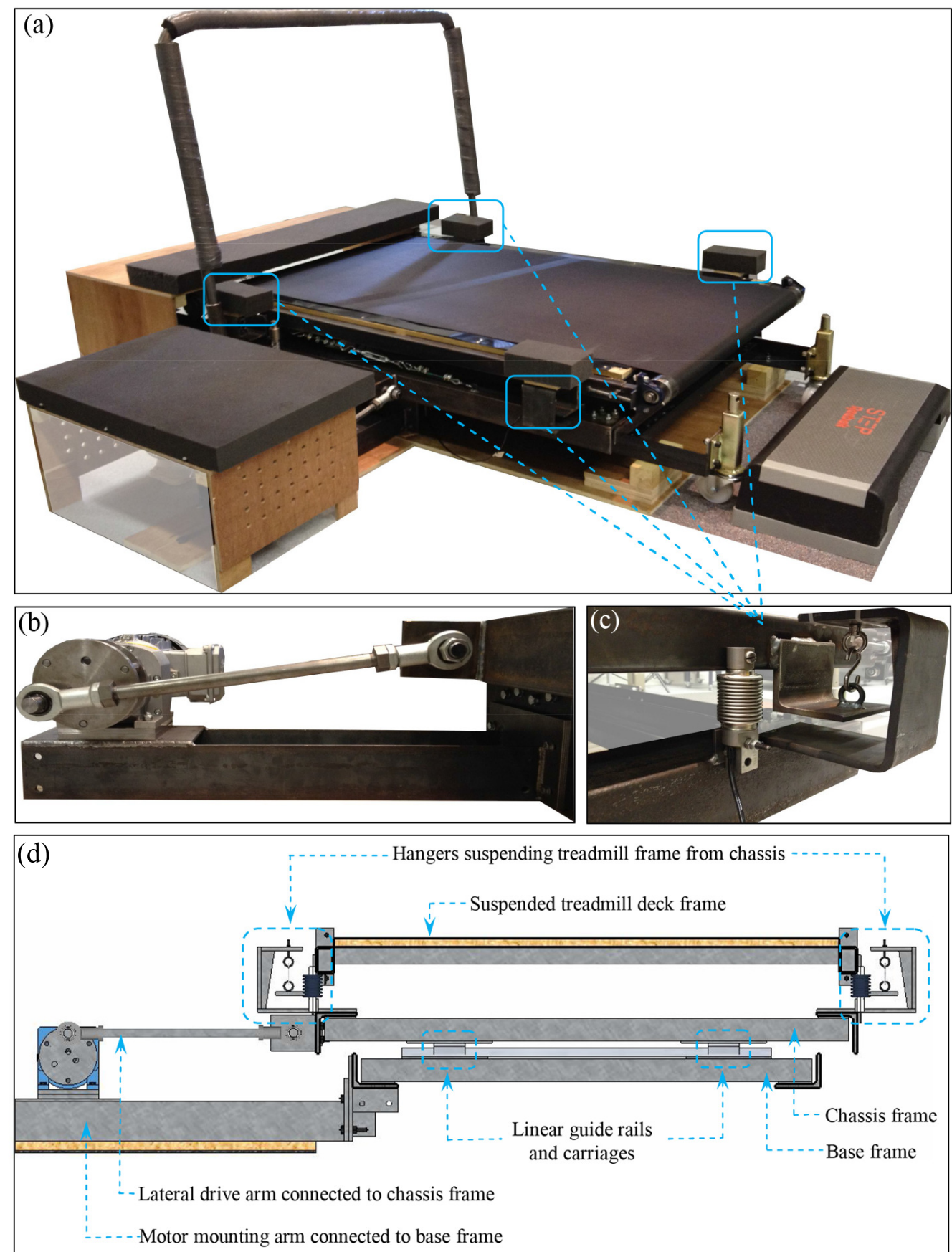

Figure 1: (a) Oscillating treadmill test rig, (b) Motor and lateral drive arm, (c) Hanger and load cell arrangement (1 of 4), the treadmill deck is supported vertically by hangers and restrained laterally by bending beam load cells, (d) Section through the test rig showing the arrangement of the base, chassis and deck frames. Reproduced with permission from [9].

The lateral GRF obtained from the treadmill rig, $F_{L, \text { meas }}$, is calculated as,

$$
F_{L, \text { meas }}=\left(\sum_{i=1}^{4} F_{c, i}\right)-m_{d} \ddot{U}_{d}
$$




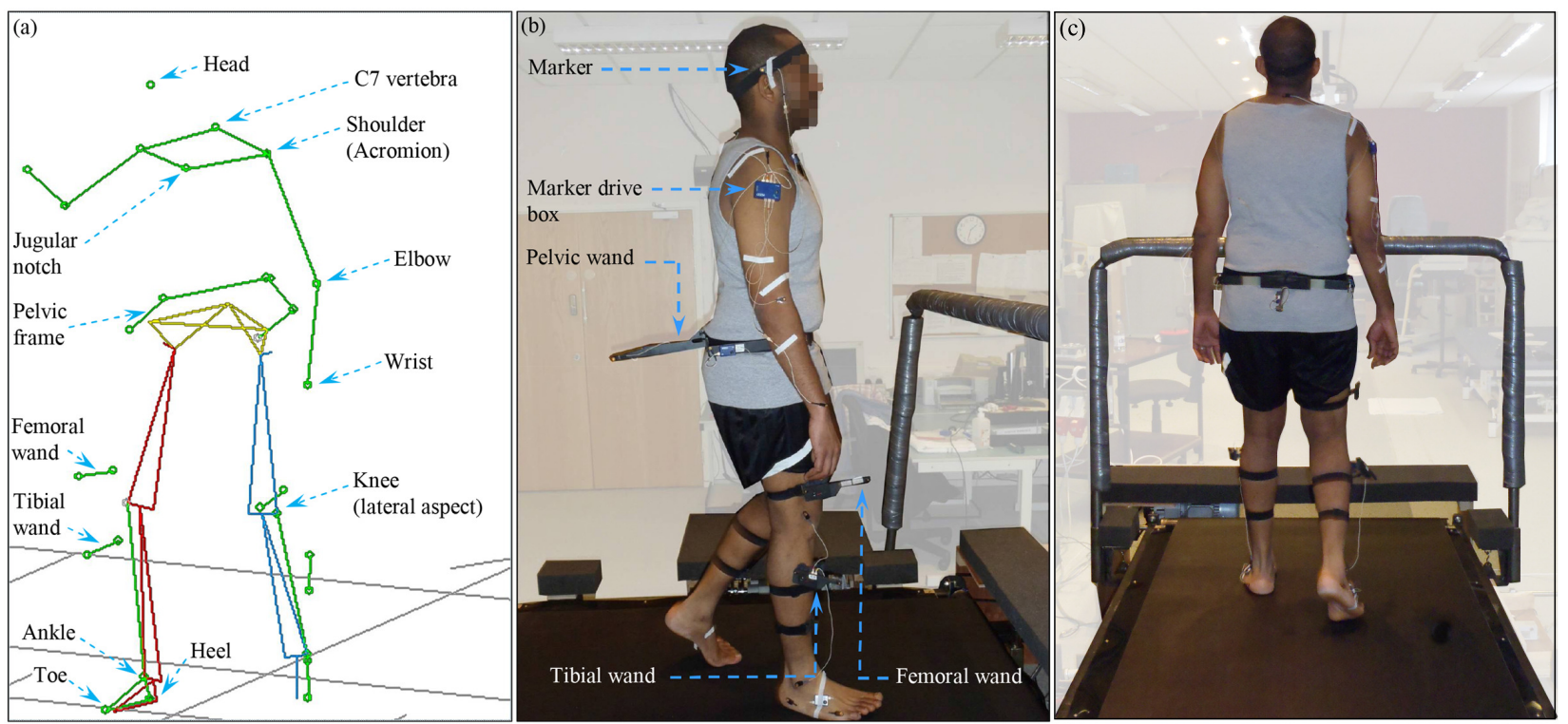

Figure 2: (a) Marker arrangement showing locations of 31 active markers, (b) and (c), Test subject instrumented with markers and gait analysis wands. (a) and (b) reproduced with permission from [9]

in which $F_{c, i}$ is the load imposed on the $i^{\text {th }}$ load cell, $m_{d}$ is the mass of the treadmill deck and associated suspended structure and $\ddot{U}_{d}$ is the lateral acceleration of the treadmill deck. $F_{L, m e a s}$ was validated against GRFs recorded on Kistler force plates (in-situ in the Human Performance Lab). Further discussion of GRF data processing and validation can be found in [9].

The raw marker data was initially low pass filtered at $6 \mathrm{~Hz}$ (BW4). Marker accelerations were obtained through double differentiation of marker displacements with the same $6 \mathrm{~Hz}$ (BW4) filter applied between each differentiation step.

\subsection{Identification of CoM location}

The number of visual markers used in this study allows a good approximation of subject CoM motion to be obtained. However, in addition to marker locations, an estimate of the distribution of subject body mass must also be made. For this purpose a model of mass distribution proposed by Zatsiorsky et al. [18] was employed. Based on a data set containing 115 subjects, Zatsiorsky et al. identify individual body segment masses (as a proportion of total subject mass) and CoM locations. Later, de Leva [19] referenced the mean CoM locations to the relevant proximal and distal joint centres, increasing the practical usability of Zatsiorsky et al's model. In this work each subject will be modelled as 15 individual body segments divided as follows: feet, shanks, thighs, pelvis, trunk consisting of abdomen and thorax regions, hands, forearms, upper arms and head. The segment masses as a percentage of overall subject mass (table 2) and segment CoM location are as per de Leva's proposal.

The lateral position of the whole-body CoM was obtained as:

$$
\mathrm{CoM}=\sum_{i=1}^{15} \frac{m_{i}}{M} \mathrm{CoM}_{i}
$$

where $m_{i}$ is the mass of body segment $i, M$ is the subject's total body mass and $\mathrm{CoM}_{i}$ is the lateral position of the CoM of segment $i$, obtained from marker data, based on de Leva's reference information. 
Table 2: Body segment masses, after [19].

\begin{tabular}{ll}
\hline Body segment & \% Body mass \\
\hline Head & 6.94 \\
Torso (Abdomen + Thorax) & 32.29 \\
Upper arm & 2.71 \\
Forearm & 1.62 \\
Hand & 0.61 \\
Pelvis & 11.17 \\
Thigh & 14.16 \\
Shank (lower leg) & 4.33 \\
Foot & 1.37 \\
\hline
\end{tabular}

\section{Linking balance behaviour with the self-excited force}

As mentioned above, the mass and damping effects of the self-excited force have previously been characterised based on experimental data obtained by Ingölfsson et al. [8]. The methodology employed in the current investigation allows a more complete picture of HSI to be obtained by virtue of the fact that body motion is recorded in parallel with the GRF generated on the oscillating deck.

An analysis of subject balance behaviour from this investigation has been presented in [9] based on a subset of data. In the following discussion the generality of the observations presented therein is established. To provide sufficient context for the following discussion, an abridged description of the key balance behaviours observed is first presented.

The following two figures, 3 and 4 , detail the response of subject 1 during individual dynamic tests. The imposed deck oscillation amplitude was $10 \mathrm{~mm}$ and deck oscillation frequencies were $0.7 \mathrm{~Hz}$ and $0.9 \mathrm{~Hz}$ respectively. In both cases the subject maintained a pacing frequency of $1.8 \mathrm{~Hz}$, resulting in a lateral forcing frequency of $0.9 \mathrm{~Hz}$ throughout the tests.

Figure 3 (a) shows the subject's CoM oscillation (solid black line and left scale), the corresponding deck oscillation is also shown (solid grey line and right scale). The underlying deck motion has been removed from all marker data, thus all reported subject motion is relative to the moving deck. Markers on the lateral aspect of the foot, at the heel and toe have been used to determine the position of the foot for each footstep (intermittent black lines), the position is calculated as the median point between both markers. The circles indicate the average foot position for the duration of the footstep.

Plot (b) shows the step widths for successive steps, defined as the lateral distance between the mean foot position marks. The x-axis position of each vertical stem is determined as the mid-time between the foot positions used to calculate that step width. Plot (c) shows the directly measured GRF, $F_{L \text {,meas }}$, and plot (d) shows its harmonic components obtained from an FFT of the filtered GRF signal.

The key feature of the subject's behaviour is the alteration of their stride width. Closer inspection of plot (a) reveals that if the subject steps to the left in time with the rig's maximum leftward displacement (and vice versa on the right), the CoM oscillation amplitude is a minimum. Conversely, when the subject steps in a direction opposite to the deck's motion and in time with the peak deck displacement, the CoM amplitude is at a maximum. This is a direct result of the sinusoidally varying equivalent inertia force experienced by the subject due to base acceleration. Due to the constant frequency relationship between the lateral forcing and deck oscillation frequencies, this results in a periodic modulation of the CoM oscillation amplitude. Coincident with this, is a narrowing and widening of the subject's gait; the lateral position of the foot is altered to maintain balance in response to the destabilising influence of deck motion. As a direct result of stride width modulation, amplitude modulation of the GRF occurs.

When the whole body behaviour responsible for the GRF is examined, it becomes apparent that the stride width and therefore the GRF is being modulated at a fixed frequency equal to the modulus of the difference between the lateral forcing frequency, $f_{\text {subject }}$ and the base oscillation frequency, $f_{\text {base }}$, in this case approximately $0.2 \mathrm{~Hz}$. Plot (d) shows the harmonic components of the GRF, the force harmonics arising due to HSI are shown in grey. Notably, these are also spaced approximately $0.2 \mathrm{~Hz}$ on either side of the 

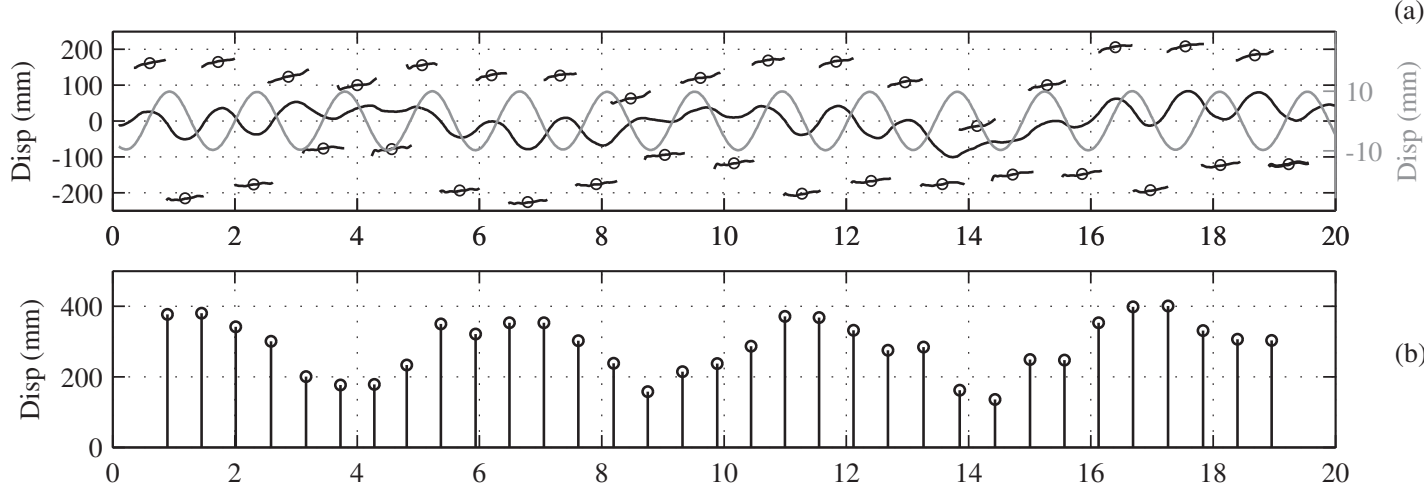

(b)
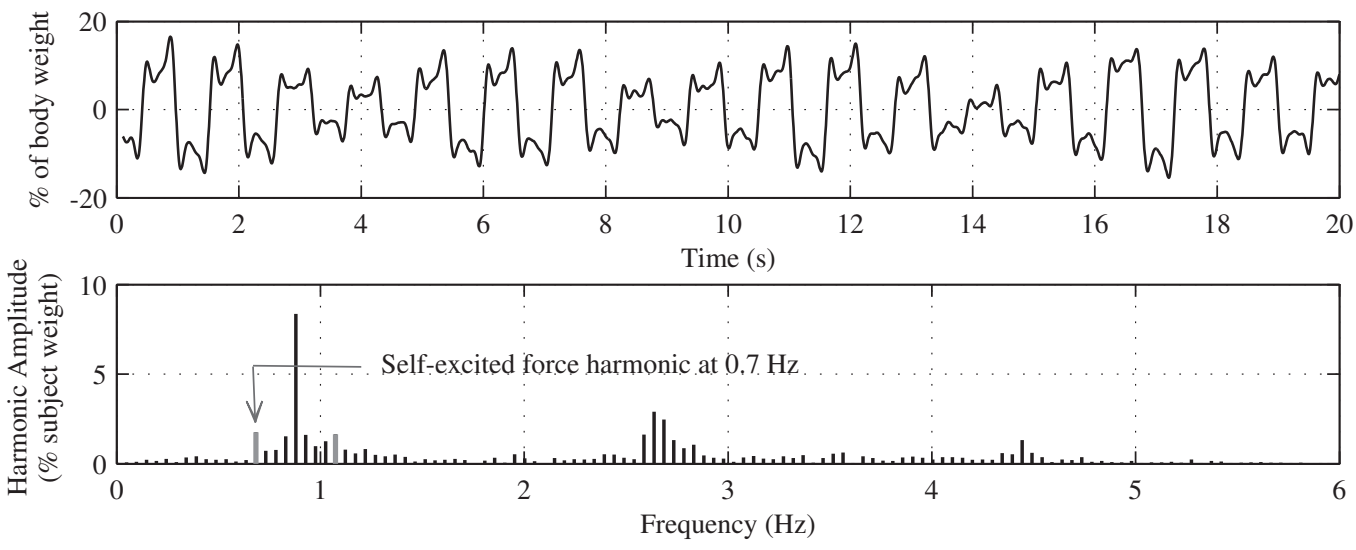

(d)

Figure 3: Deck oscillation amplitude $=10 \mathrm{~mm}$, frequency $=0.7 \mathrm{~Hz}$. (a) CoM oscillation (continuous black line and left scale), deck oscillation (continuous grey line and right scale) and lateral foot position (intermittent black line and left scale), (b) step widths, (c) directly measured GRF, (d) harmonic components of the directly measured GRF (interaction forces shown in grey). Reproduced with permission from [9].

fundamental force harmonic, a measure predicted by the biomechanical behaviour described above. This is in agreement with theoretical predictions in [10], obtained using the inverted pendulum biomechanical model. The proximity of the interaction harmonics to $f_{\text {subject }}$ is consistent with their identification as frequency sidebands resulting from amplitude modulation of the GRF. In signal processing terminology, the GRF produced in the absence of base motion may be considered a 'carrier' wave. The pedestrian's balance response modulates this wave form, resulting in frequency sidebands appearing in the modulated wave's spectrum. Due to the fact that the modulating frequency is given by $\left|f_{\text {base }}-f_{\text {subject }}\right|$, one sideband will always coincide with the structure's oscillation frequency.

In Fig. 4, the base oscillation amplitude is $10 \mathrm{~mm}$ but the frequency is increased to $0.9 \mathrm{~Hz}$, synchronised with the lateral forcing frequency imposed by the subject. As may be expected based on the preceding discussion, the alterations in stride width previously observed are absent, plot (a) and (b). CoM oscillation amplitude, stride width and GRF amplitude all remain relatively stable during the test. Due to the constant phase relationship between the subject's lateral forcing and the deck oscillation frequencies, the magnitude of the equivalent inertia force imposed on the subject at the time of foot placement, remains the same from gait cycle to gait cycle. This eliminates the need to periodically alter foot placement position to maintain frontal plane stability; instead, the subject adopts a wider gait, sufficient to deal with the deck-induced inertia force, leading to a scale increase in the GRF.

Recognising the significance of amplitude modulation in generating the self-excited force, the percentage modulation depth is defined allowing the degree of amplitude modulation to be quantified; $0 \%$ modulation corresponds to a waveform with constant amplitude while $100 \%$ modulation corresponds to a waveform 

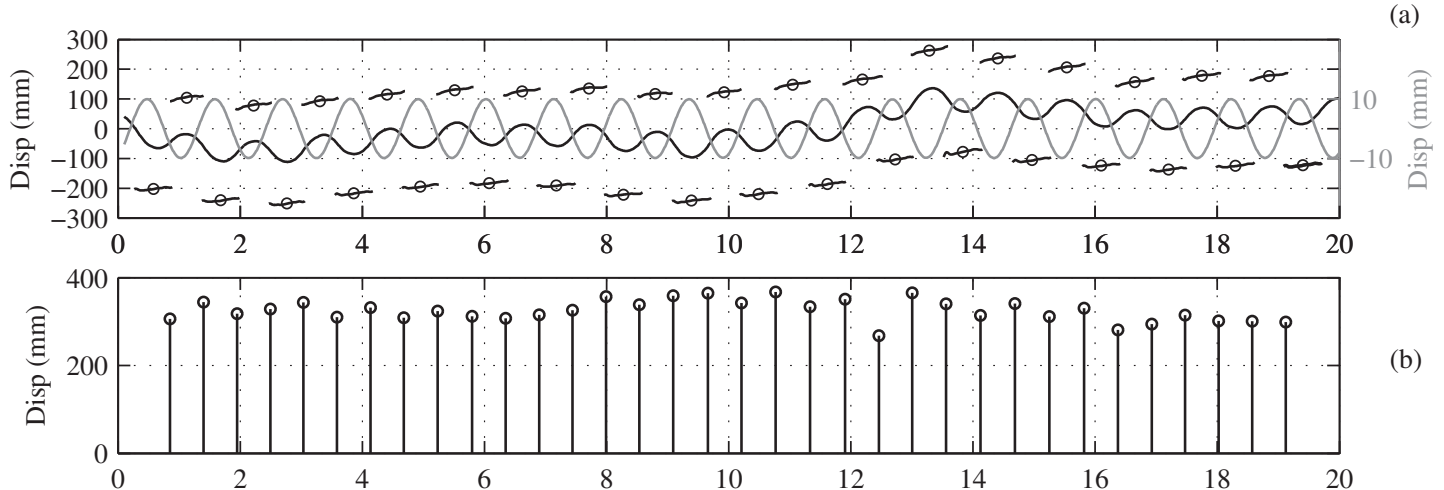

(b)
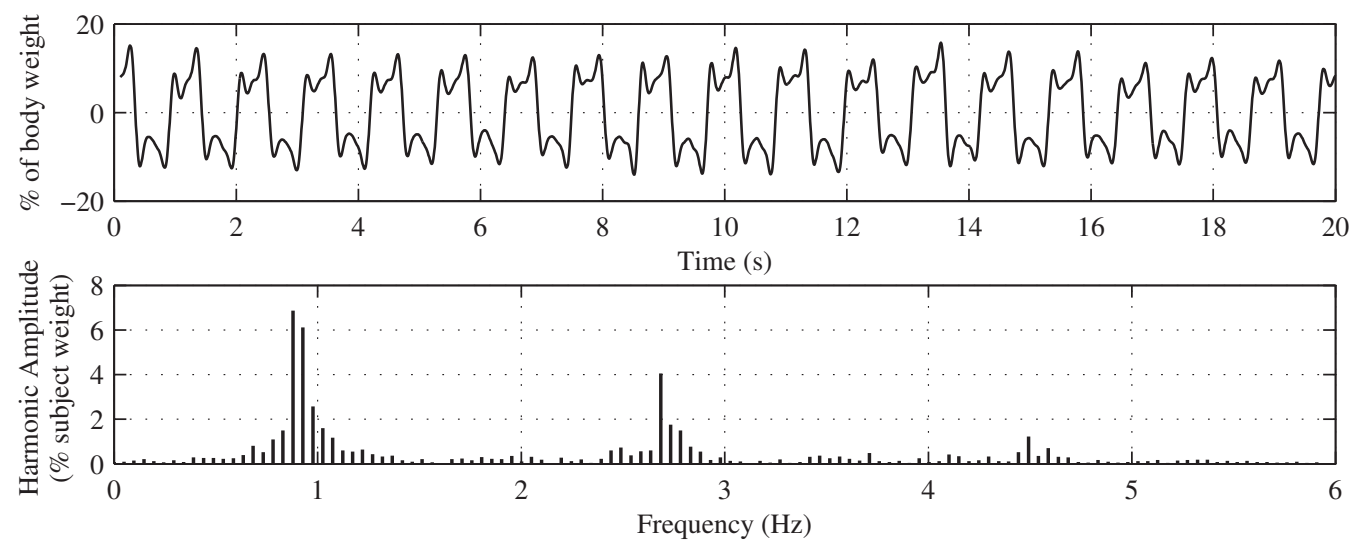

(d)

Figure 4: Deck oscillation amplitude $=10 \mathrm{~mm}$, frequency $=0.9 \mathrm{~Hz}$. (a) CoM oscillation (continuous black line and left scale), deck oscillation (continuous grey line and right scale) and lateral foot position (intermittent black line and left scale), (b) step widths, (c) directly measured GRF, (d) harmonic components of the directly measured GRF. Reproduced with permission from $[9]$.

whose amplitude periodically reduces to zero (at the modulation frequency). MD is given by:

$$
\mathrm{MD}=100 \times \frac{\text { wave peak }- \text { wave trough }}{\text { wave peak }+ \text { wave trough }}
$$

where the wave peak was determined by discarding the maximum value of stride width and obtaining the average of the next two largest values. The corresponding procedure was applied to the minimum stride widths to determine the wave trough. In the case of Fig. 3, MD $=42 \%$

The percentage MD and normalised average stride width have been determined for each test completed by subject 1 , Fig. 5 . The average stride width has been normalised by the average obtained for the subject during tests in which no lateral deck motion was imposed.

During $5 \mathrm{~mm}$ amplitude tests, Fig. 5 (a), MD is relatively low $(<20 \%)$, although this increases as the deck oscillation frequency increases imposing higher amplitude accelerations on the subject. Interestingly, despite the low oscillation amplitude, the mean stride width is approximately $50 \%$ greater than that observed during tests in which no deck motion was imposed. This underlines the subject's sensitivity to deck motion in the frontal plane.

During larger amplitude oscillations, MD increases and is generally in excess of $30 \%$ and in some cases $40 \%$. The stride width ratio remains stable at approximately 1.5 for $5 \mathrm{~mm}, 10 \mathrm{~mm}$ and $20 \mathrm{~mm}$ tests. However it increases significantly for $35 \mathrm{~mm}$ and $50 \mathrm{~mm}$ tests.

The stabilisation of stride width discussed previously is clearly seen for cases when the subject is synchronised with the deck. In all cases when $\left(f_{\text {base }} / f_{\text {subject }}\right) \approx 1$, MD is significantly reduced. In such cases the average stride width is typically wider than for unsynchronised walking. 

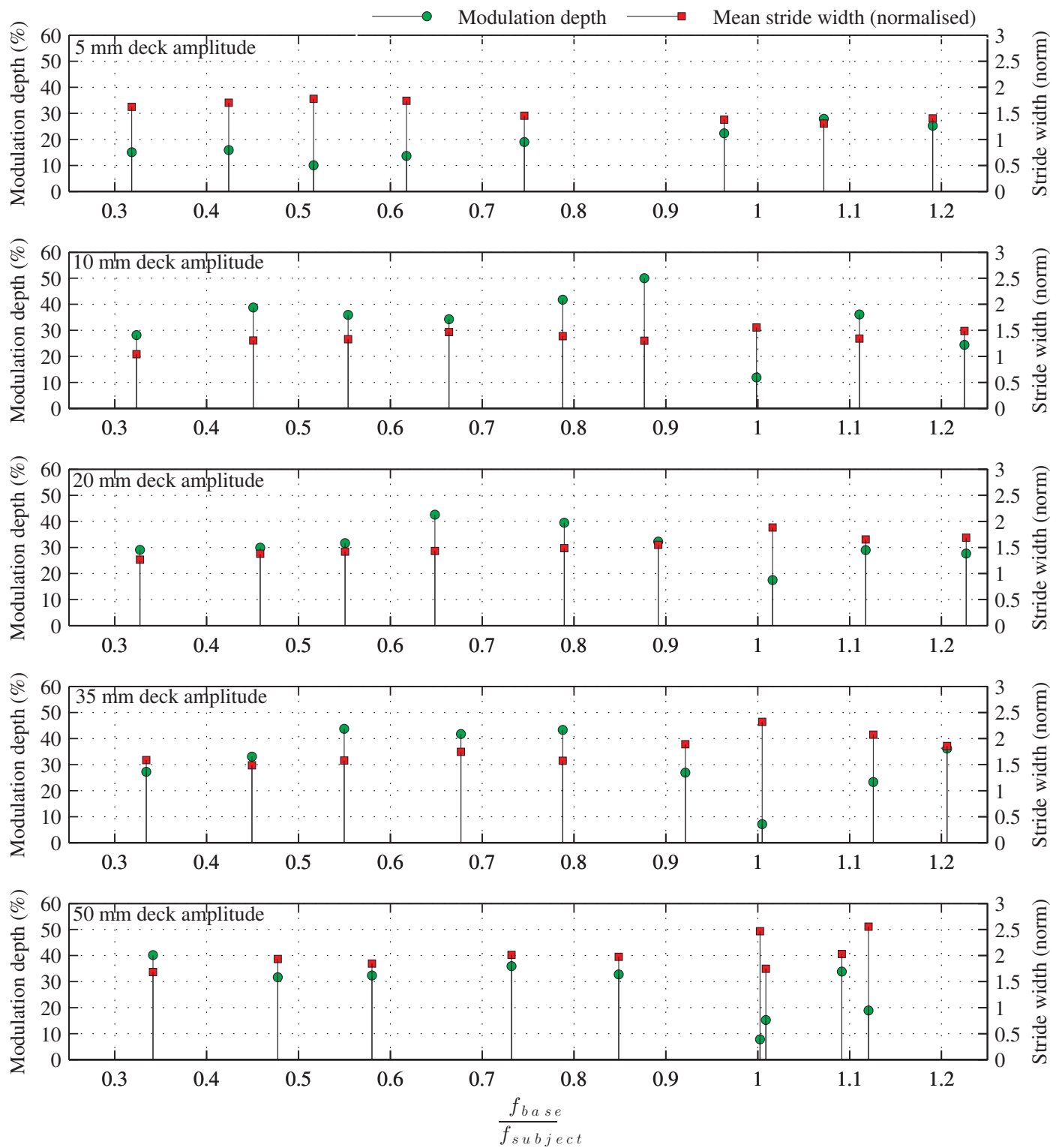

Figure 5: The percentage MD and normalised average stride width for each test completed by subject 1 . Data shown for 5 $\mathrm{mm}$ (a), $10 \mathrm{~mm}$ (b), $20 \mathrm{~mm}$ (c), $35 \mathrm{~mm}$ (d) and $50 \mathrm{~mm}$ (e) amplitude tests, [20].

These analyses have been repeated for the remaining 9 subjects and average values of MD, Fig. 6 and normalised mean stride width, Fig. 7 , have been determined. The data has been sorted into frequency bins arranged as follows: Bins $=[(0.25-0.35),(0.36-0.45),(0.46-0.55),(0.56-0.65),(0.66-0.75),(0.76-$ $0.85),(0.86-0.95),(0.96-1.05),(1.06-1.15),(1.16-1.25)] \mathrm{Hz}$. The mean and standard deviation of data points in each bin was then determined. The mean frequency ratio in each bin is used to determine the $\mathrm{x}$-axis position of each data point in Figs. 6 and 7 .

The population average data for MD is in agreement with that presented for subject 1 . For $5 \mathrm{~mm}$ amplitude tests, MD is approximately $20 \%$ with very little inter-subject variability. However as deck acceleration amplitude increases, so too does MD. The tendency for stride widths to stabilise at a constant value and therefore reduce MD during synchronisation is also visible in the population data. Figure 7 also clearly demonstrated the tendency for mean stride width to increase with deck displacement amplitude 

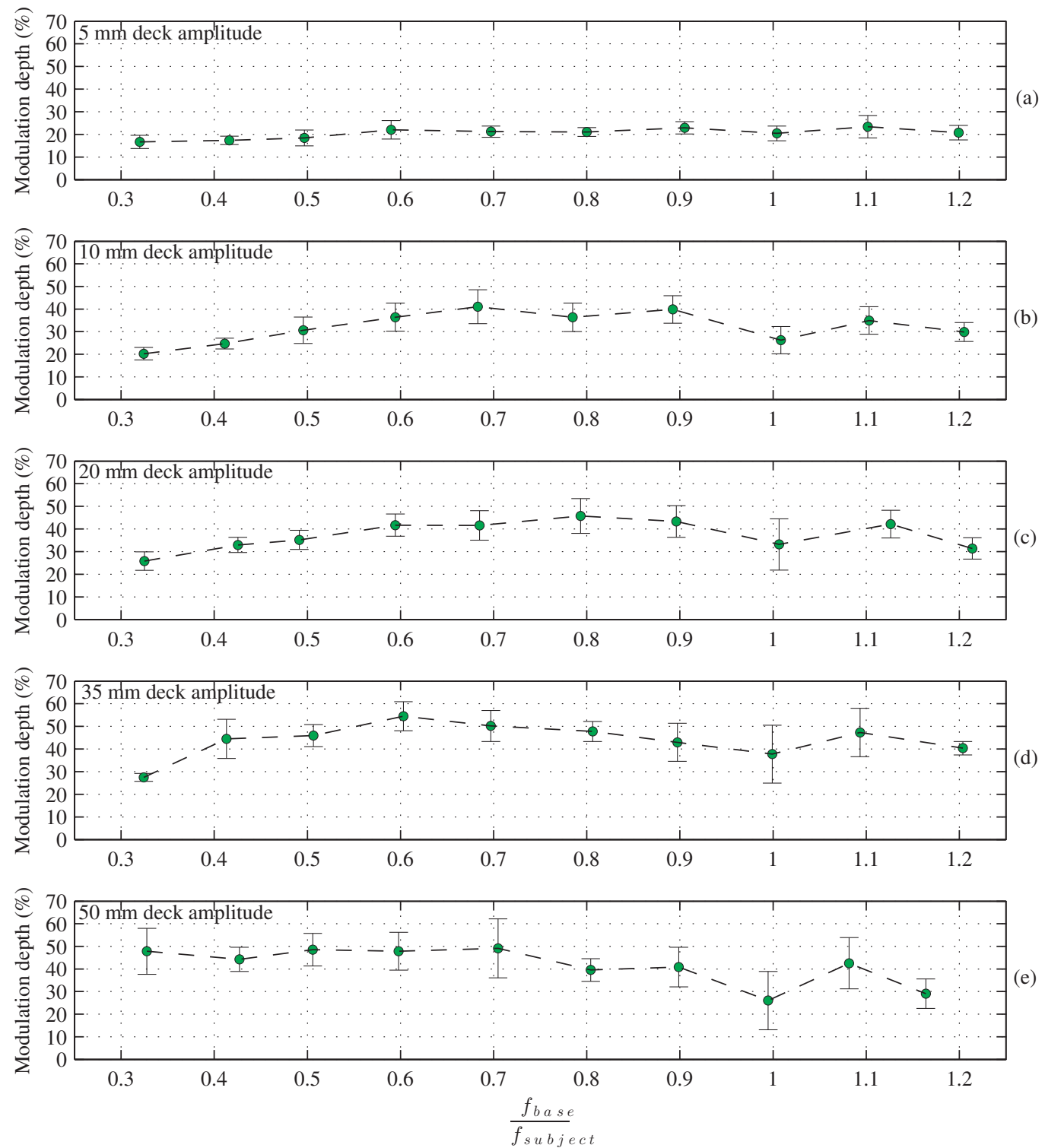

Figure 6: The percentage MD for 10 subjects. The mean \pm 0.5 standard deviations (i.e. full bar height $=1$ SD) is plotted for each frequency bin within (a) $5 \mathrm{~mm}$, (b) $10 \mathrm{~mm}$, (c) $20 \mathrm{~mm}$, (d) $35 \mathrm{~mm}$ and (e) $50 \mathrm{~mm}$ amplitude tests, [20].

and frequency. This behaviour is conveniently visualised by plotting normalised stride width against deck velocity amplitude, Fig. 8. In doing so, an approximately linear relationship is identified. Despite intersubject variability increasing as deck acceleration amplitude grows, it is reasonable to conclude that the biomechanical behaviour identified in figures 3 and 4 is representative of the test population's response to lateral base motion.

To investigate the frequency of stride width modulation, the modulation frequency ratio, $\beta$, is defined as the ratio of modulation frequency observed to that predicted by the the base oscillation and forcing frequency relationship, $\left|f_{\text {base }}-f_{\text {subject }}\right|$ :

$$
\beta=\frac{\text { Observed modulation frequency }}{\left|f_{\text {base }}-f_{\text {subject }}\right|}
$$



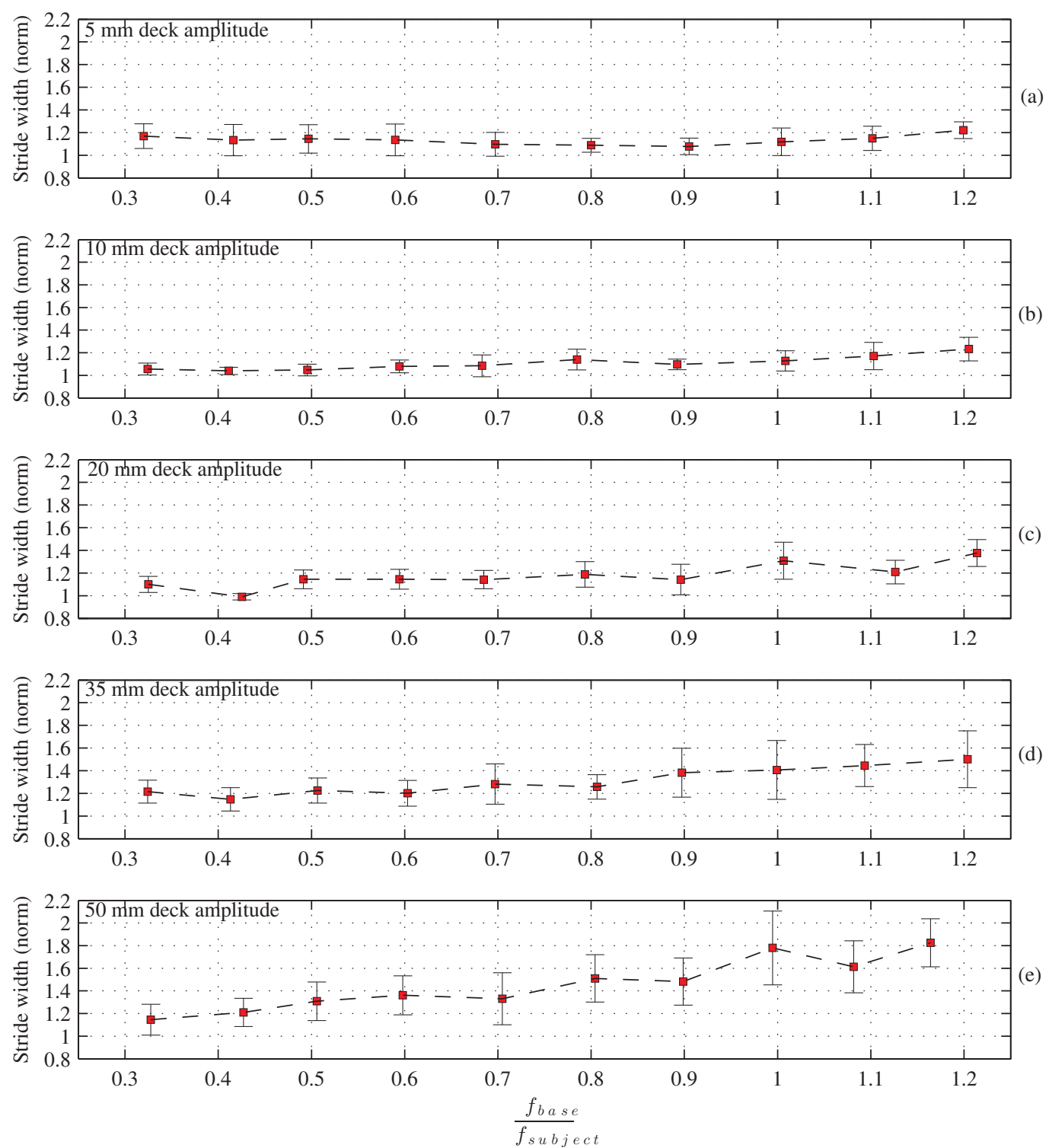

Figure 7: The mean normalised stride width for 10 subjects. The mean \pm 0.5 standard deviations (i.e. full bar height $=1$ SD) is plotted for each frequency bin within (a) $5 \mathrm{~mm}$, (b) $10 \mathrm{~mm}$, (c) $20 \mathrm{~mm}$, (d) $35 \mathrm{~mm}$ and (e) $50 \mathrm{~mm}$ amplitude tests, [20]. 


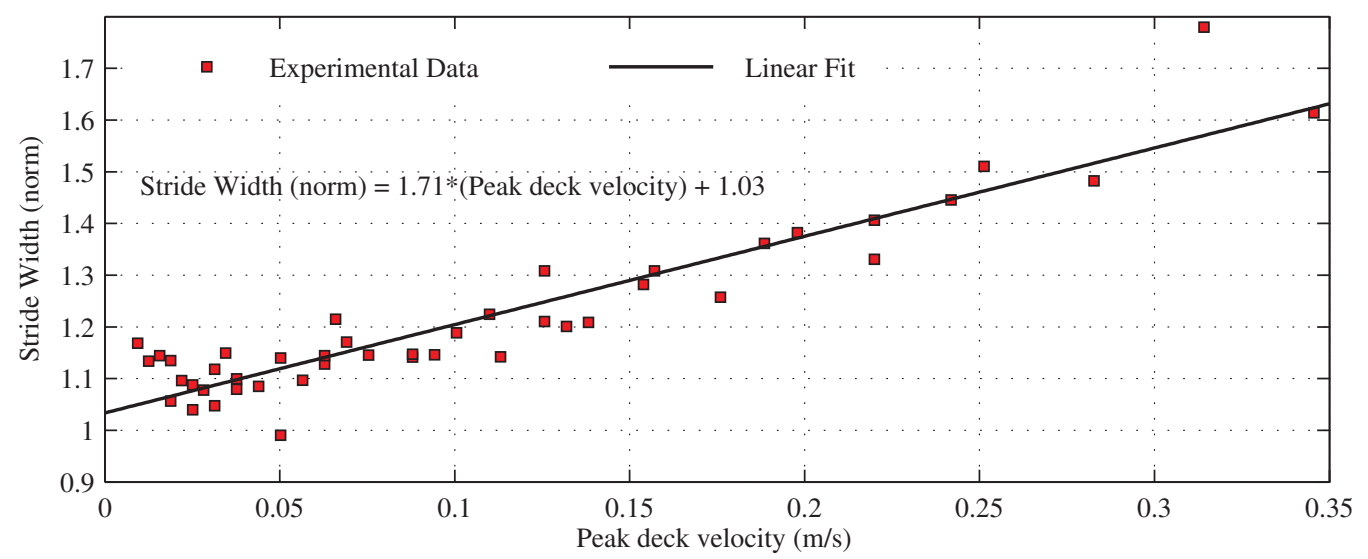

Figure 8: The mean normalised stride width plotted against peak deck velocity, identifying an approximately linear relationship.

If $\beta=1$ across the full test matrix, it can be concluded that the experimental modulation frequency is given by the expression $\left|f_{\text {base }}-f_{\text {subject }}\right|$ and is therefore a direct result of the frequency relationship between lateral forcing and deck oscillation frequencies. One can therefore logically conclude that the previously described motivation for stride width modulation (to maintain lateral stability) is consistent across all tests, rather than being a chance observation in the tests selected for discussion.

Figure 9 show the ratio $\beta$ calculated for each test completed by subject 1 . Note that $\beta$ values for which $f_{\text {base }} \approx f_{\text {subject }}$ have been omitted as the parameter is not applicable under this frequency regime.

It can be seen that in the majority of cases $\beta \approx 1$, indicating that periodic stride width modulation is generally occurring at a frequency equal to $\left|f_{\text {base }}-f_{\text {subject }}\right|$. The biomechanical motivation for alteration of foot position has been identified as being a response to a sinusoidally varying inertia force. Furthermore, the frequency of the SE force harmonic is also identified as $f_{\text {subject }} \pm\left|f_{\text {base }}-f_{\text {subject }}\right|$. Therefore, Fig. 9, in which $\beta$ is predominantly equal to one, establishes the link between pedestrian balance behaviour and the generation of a SE force harmonic.

Note that for the lowest frequency test during $5 \mathrm{~mm}$ and $10 \mathrm{~mm}$ amplitude oscillations, plots (a) and (b) in Fig. $9, \beta \approx 0$. This is due to the stride width modulation not being sufficiently well defined for a dominant harmonic to be extracted. In both cases the peak lateral acceleration experienced by the subject $<0.04 \mathrm{~m} / \mathrm{s}^{2}$ and resulted in negligible influence on stride width. However as the strength of external stimulus grows, periodic modulation is quickly established. Although not shown here, the same trend $(\beta \approx 1)$ was demonstrated for all subjects who took part in the campaign.

Figures 5 to 9 demonstrate that the detailed discussion presented in relation to figures 3 and 4 describes typical gait behaviour for the test population. While the underling mechanism at the core of lateral human-structure interaction have been identified, the significance of inter-subject variability must also be acknowledged. All subjects exhibit stride width modulation in response to deck motion, however the degree to which individuals are influenced and therefore the strength of the interaction show large inter-subject variability. Therefore there is much work to be done identifying and correlating key parameters with quantitative measures of the interaction mechanism.

\section{The inverted pendulum model of frontal plane balance}

The work of Macdonald [10], itself a development of Barker's earlier work, [21], has motivated a number of researchers $[14,15,22]$ to pursue a biomechanics-centric analysis of HSI. This approach typically utilises the inverted pendulum (IP) as a means of approximating human frontal plane CoM motion during locomotion, Fig. 10. Application of this simple biomechanical model, stabilised via active control of support or foot placement has produced promising results thus far. However, there are some outstanding uncertainties 

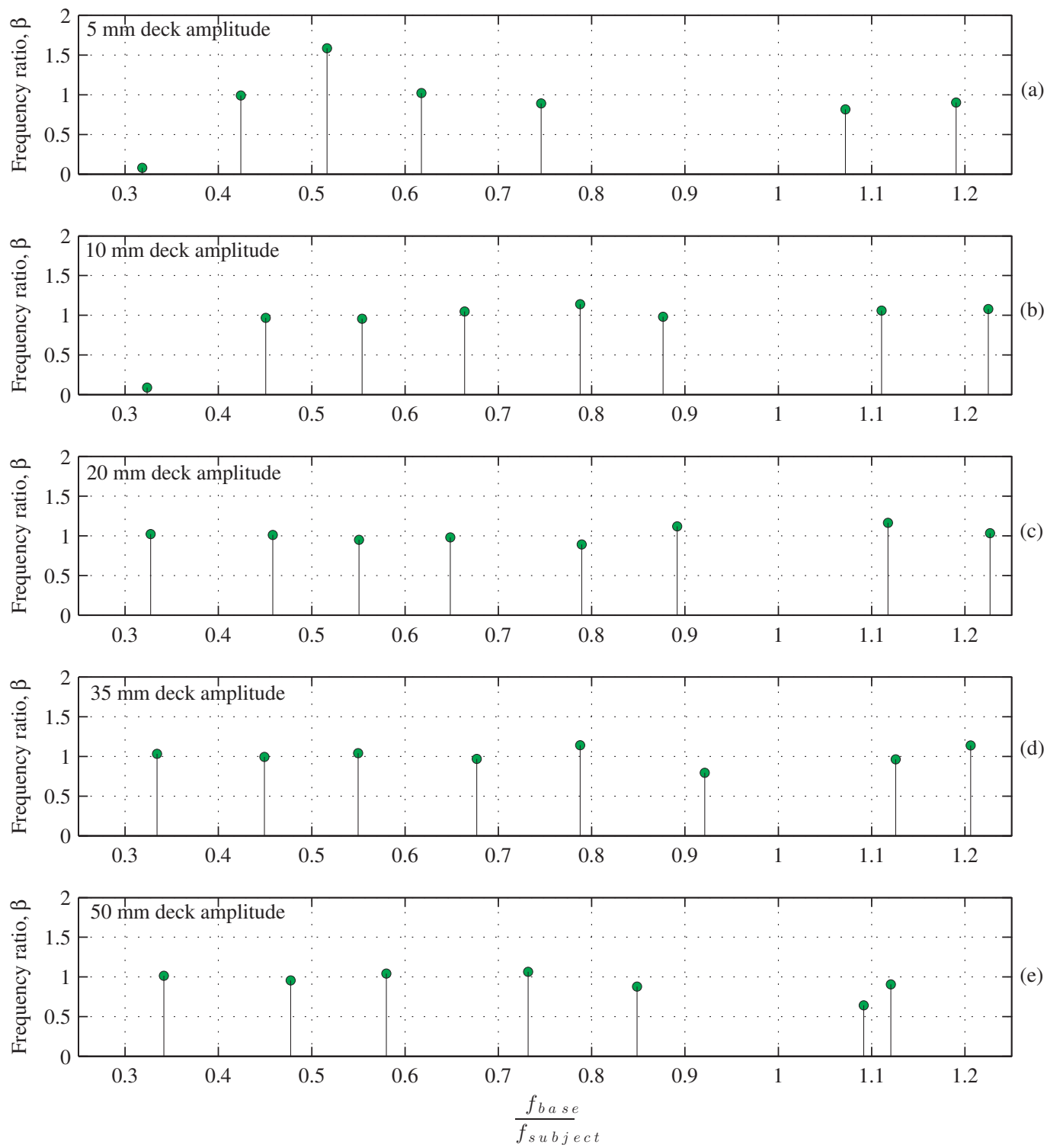

Figure 9: The ratio of observed stride width modulation frequency to that predicted by the expression, $\left|f_{\text {base }}-f_{\text {subject }}\right|$. Data shown for $5 \mathrm{~mm}$ (a), $10 \mathrm{~mm}$ (b), $20 \mathrm{~mm}$ (c), $35 \mathrm{~mm}$ (d) and $50 \mathrm{~mm}$ (e) amplitude tests, completed by test subject 1, [20].

regarding its suitability for the specific case of walking on a laterally oscillating structure. The aim of the following analysis is to address this uncertainty.

The IP model is well established in the biomechanics field [23]. The model, coupled with the balance law proposed in [11] can be considered a semi-active model by virtue of the fact that discrete balance corrections are made at the time of support placement; the so-called foot placement balance strategy. While simulating the single stance phase, motion of the pendulum mass is defined solely by its initial conditions and passive mechanics. If one considers walking on a stationary surface, foot placement has been shown to be the main balance strategy, with minor corrections provided by rotation of the upper body about the supporting ankle and hip [12]. Therefore the semi-active inverted pendulum model stabilised through foot placement is suitable.

Walking on a laterally moving bridge is fundamentally different from walking on stationary ground. In 


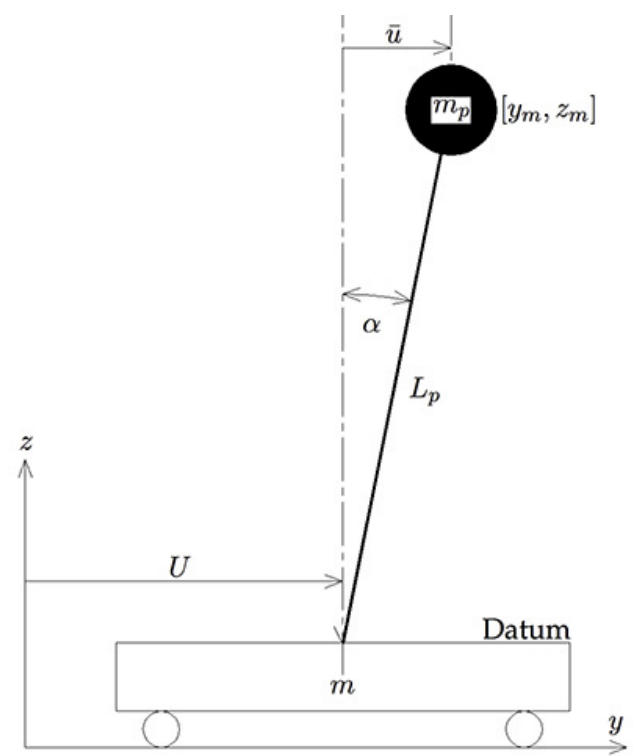

Figure 10: Inverted pendulum supported on a structure free to move laterally, indicated by coordinate $U$. The coordinate $y_{m}$ approximates the lateral or frontal plane motion of a human during the single stance gait phase. The lateral distance between pendulum mass and support positions is denoted by $\bar{u}$ while the pendulum length is $L_{p},[20]$.

this case a pedestrian must decide where to place their foot based only on the sensory information they have at the instant the foot is placed. However, during the single stance phase the bridge continues to oscillate, providing additional sensory stimulus potentially altering the feeling or sense of stability.

On this basis it is logical to hypothesise that external perturbations imposed by a swaying bridge may be corrected for through some form of active control implemented during the single stance phase. Essentially, pedestrians may alter their CoM motion in response to a continuously changing lateral inertia force, in order to maintain stability. The CoM motion observed while subjects walked on the laterally oscillating treadmill provide a benchmark against which to compare IP model predictions. The objective is thus to determine if the passive dynamics of the IP model are sufficient to describe human CoM motion while walking on an oscillating deck, or if some form of active motion control should also be implemented.

Lateral acceleration of the IP is approximated by the linearised equation of motion,

$$
\ddot{y}_{m}=-\ddot{U}+\omega^{2} \bar{u}
$$

where $\ddot{U}$ is the lateral acceleration of the supporting structure, $\omega$ is the natural frequency of an equivalent hanging pendulum and $\bar{u}$ is the lateral eccentricity of the pendulum root with reference to the pendulum mass [10]. By ensuring that the pendulum support is placed at a sufficient eccentricity $\bar{u}$, for each step, a 
stable gait is simulated. The eccentricity of the support is obtained from the expression,

$$
\bar{u} \geq \frac{\dot{y}_{m, 0}}{\omega} \pm b_{\min }
$$

where $\dot{y}_{m, 0}$ is the pendulum velocity at the time the IP support is placed and $b_{m i n}$ is an additional stability margin, the sign of which alternates on successive steps $[11,12]$. The quantity $y_{m}+\frac{\dot{y}_{m}}{\omega}$ is defined as the extrapolated CoM or XCoM as it considers the extrapolated trajectory of the CoM based on its current velocity. For a further discussion of the simulation process, the reader is referred to [10].

\subsection{Pendulum versus subject CoM motion: stationary deck}

A comparison between observed subject behaviour and model output is now considered. Analysis of data relating to subject number 4 is discussed before summary data for the full test population is presented. To facilitate the comparison, an IP model corresponding to the subject in question was established. The pendulum length was determined as the vertical distance to the subject's CoM (based on marker data and de Leva's model [19]), while the IP mass was equal to the subject's body mass. The pendulum was first tuned to match the subject's observed behaviour during a stationary test in which no treadmill oscillation was imposed. Pendulum initial position and velocity were chosen to match that of the subject's CoM at the beginning of the comparison. To estimate the initial pendulum support position, a constant medial offset of $35 \mathrm{~mm}$ was applied to the lateral position of the foot-mounted markers. This approximates the average position of the subject's centre of pressure $(\mathrm{CoP})$.

In order to further tune IP behaviour to the subject, the minimum stability margin, $b_{\min }$ from Eq. (6) was used as a tuning parameter. The cumulative area under the (absolute) GRF time history was compared for the IP and subject. The areas were compared based on a signal comparison lasting approximately $18 \mathrm{~s}$. $b_{\min }$ was then altered until the difference in areas was a minimum. The final value selected for $b_{\text {min }}$ in the case presented below was $0.026 \mathrm{~m}$ resulting in an area difference of $1.1 \%$. Figure 11 shows the result of this tuning process for CoM position, velocity and acceleration, plots (a) to (c). Plot (d) shows a comparison between XCoM and CoP position for the subject and IP.

The initial drift in IP trajectory results from a discrepancy between the IP and subject's initial CoP position. This results from the approximation of CoP position outlined above. The influence of this discrepancy is dissipated within the first two steps and has no bearing on the comparison thereafter. The mean value of both time-histories (determined after the first two steps have occurred) has been removed from both sets of data in plot (a). This removes the influence of the initial drift in simulated CoM motion. The same offsets have also been removed from the simulated and observed data in plot (d). The remaining drift in IP position seen in plot (a) results from small variations in IP step duration, matching the intra-subject variability exhibited in the experimental data.

Considering the CoM position, velocity and acceleration comparisons in plots (a), (b) and (c), the IP appears to simulate the CoM trajectory quite well, particularly in the case of the CoM velocity, plot (b). With reference to plot (a), it is apparent that the IP model is unable to simulate 'atypical' CoM oscillations such as arise from the subject drifting across the treadmill deck as they walk. Nevertheless, the general character of the simulated oscillatory behaviour is in good agreement with that experimentally observed. The CoM accelerations in plot (c) show good amplitude agreement (ensured by the $b_{\text {min }}$ tuning process), although the subject's acceleration during the mid-stance deviates significantly from the smooth trajectory simulated by the IP.

From this comparison it is apparent that both the low and high frequency content of the CoM motion is not recreated by the IP model. This can be seen, for low frequency content, in plot (a) in which the drifting of the subject's CoM is not captured by the model. Inability to simulate high frequency content can be seen in plot (c) in which the subject's CoM acceleration shows high frequency motion during the single stance phase. This low and high frequency motion has a negligible impact on the generation of SE forces, discussed in section 4; as such, it can be considered only a minor deficiency in the model.

Plotting the simulated versus observed behaviour for position, velocity and acceleration data, the correlation coefficient $(\mathrm{CC})$ is used as a quantitative measure of the linearity between simulated and observed 

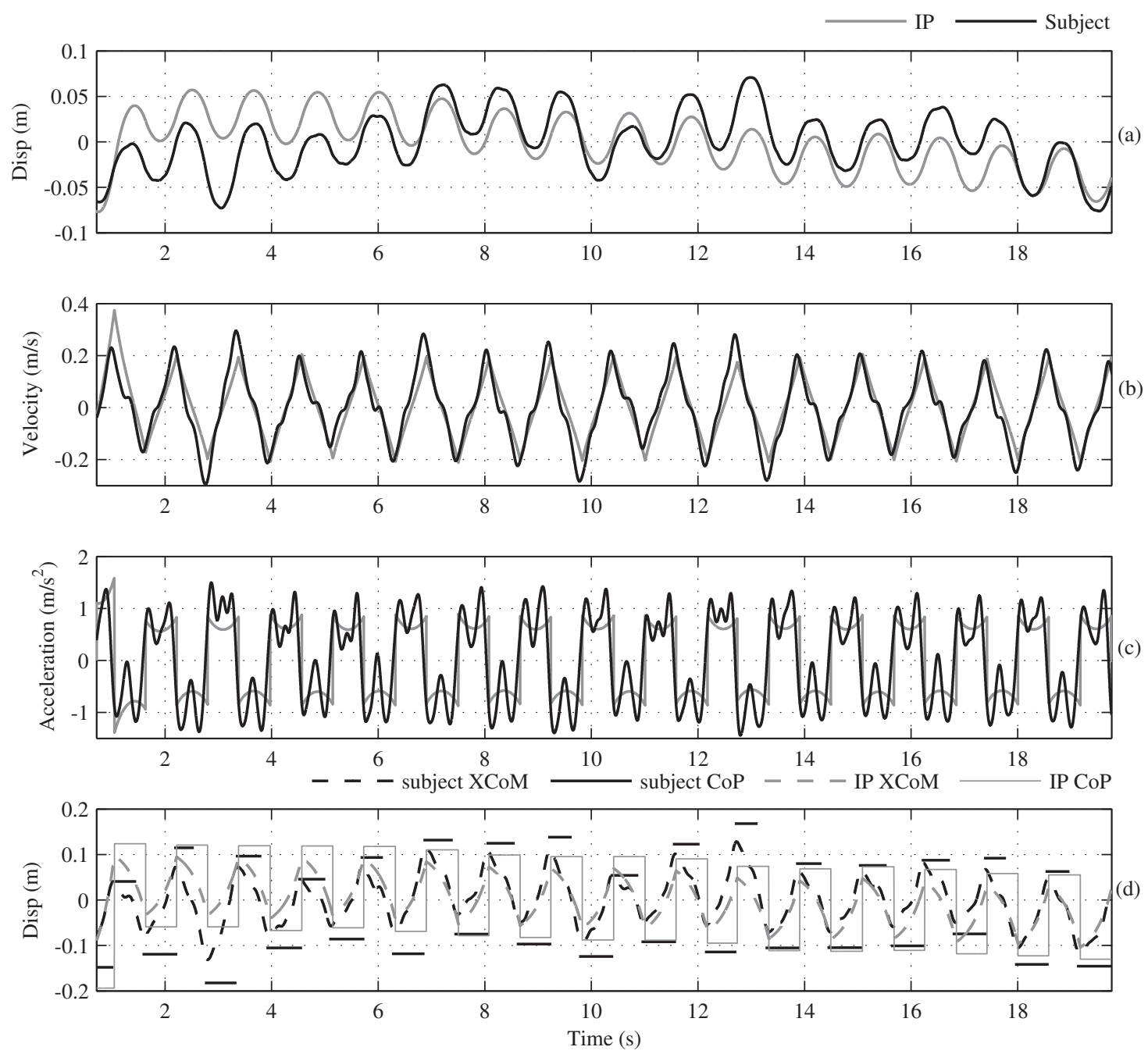

Figure 11: Comparison between subject and equivalent IP, (a) CoM position (correlation coefficient, CC $=0.55$, slope of the linear regression line, $\left.\mathrm{m}_{r e g}=0.51\right)$, (b) CoM velocity $\left(\mathrm{CC} / \mathrm{m}_{r e g}=0.93 / 0.77\right),(\mathrm{c}) \mathrm{CoM}$ acceleration $\left(\mathrm{CC} / \mathrm{m}_{r e g}=0.81 / 0.66\right)$ and (d) XCoM and CoP position. A constant medial offset of $35 \mathrm{~mm}$ has been applied to the recorded foot position to approximate the average position of the subject's CoP [20].

behaviour. In this way, linearity is used as the measure of similarity. These were calculated as $0.55,0.93$ and 0.81 for position, velocity and acceleration respectively. This parameter is independent of any scaling difference between simulated and observed trajectories. This is quantified by the slope of the line of linear regression, $\mathrm{m}_{\text {reg }}$, between observed and simulated behaviour. These are calculated as 0.51, 0.77 and 0.66, all identifying that the magnitude of position, velocity and acceleration is underestimated by the IP model.

Plot (d) shows the XCoM and CoP positions for the subject and IP. Note that the subject's CoP was approximated as described above. Again, with the exception of anomalous steps, eg. at $\mathrm{t}=[3,5,13] \mathrm{s}$, the IP approximates the subject's behaviour well. Considering the complexity of human locomotion and the comparative simplicity of the biomechanical model, the general agreement seen in Fig. 11 is, in the authors opinion, remarkable. 


\subsection{Pendulum versus subject CoM motion: oscillating deck}

Having tuned the IP model to the specific subject under consideration, the comparison is now repeated for dynamic tests (while employing the same value of the tuning parameter $b_{\min }$ ). If the model performs well, it can be concluded that a passive IP is sufficient to describe CoM motion and its continued use is justified. Assessing how well the IP model approximates the subject's behaviour under these dynamic conditions is essential if the model is to be applied with confidence in the simulation of crowd-induced vibration. What follows in figures 12, 13 and 14 is a selection of dynamic test comparisons which indicate typical IP model performance. In all four cases the subject's lateral forcing frequency was approximately $0.9 \mathrm{~Hz}$.

In order to determine IP support placement, Eq. (6) requires the pendulum velocity at the time of support placement, $\dot{y}_{m, 0}$. When the IP is supported on a moving deck, there are two possible velocities that may be used within the balance law, velocity relative to the moving deck or velocity measured in a global stationary reference frame. With reference to Figs. 12 to 14, when determining the IP support placement position from Eq. (6), the CoM velocity relative to the moving deck was found to provide the closest approximation of subject behaviour.

Figure 12 represents the IP/subject comparison during lateral oscillations of $5 \mathrm{~mm}$ amplitude at a frequency of $0.3 \mathrm{~Hz}$, the lowest magnitude acceleration imposed. Despite the low acceleration magnitude $\left(0.018 \mathrm{~m} / \mathrm{s}^{2}\right)$, there is an immediate widening of the subject's gait which is not predicted by the CoP placement law, Fig. 12 (d). This is accompanied by larger amplitude CoM oscillations, plot (a), with a corresponding increase in velocity and acceleration amplitude, plots (b) and (c). This abrupt change in subject stride width, initially identified in Fig. 8, is surprising considering the relatively low magnitude of deck motion imposed. The CCs were determined as $0.49,0.95$ and 0.86 for position, velocity and acceleration respectively. These values are broadly in line with the static test, however the disparity between observation and simulation is confirmed by the $\mathrm{m}_{\text {reg }}$ values, 0.41 (pos), 0.57 (vel) and 0.54 (acc). These parameters reveal that the visually reduced agreement results from scaling differences due to the subject's wider gait, rather than any fundamentally different oscillatory behaviour. Indeed, the CCs for velocity and acceleration are marginally higher than those obtained during the static test

Figure 13 shows the comparison during lateral oscillations of $10 \mathrm{~mm}$ amplitude at a frequency of $0.5 \mathrm{~Hz}$. The passive dynamics of the IP appear to predict reasonably well the subject's oscillatory behaviour in the face of this strengthening stimulus. This is particularly the case between $4 s<=t<=12 \mathrm{~s}$, during which position, velocity and acceleration are well predicted. However atypical behaviour, eg. between $13 \mathrm{~s}<=$ $t<=14 \mathrm{~s}$ obviously cannot be predicted by the IP. The correlation and regression data was calculated as $\mathrm{CC} / \mathrm{m}_{\text {reg }}=0.64 / 0.34$ (pos), 0.93/0.66 (vel) and 0.81/0.59 (acc). Both velocity and acceleration comparison show similar parameter values to the tests discussed previously, however the position CC is noticeably improved, although significantly underestimated in terms of amplitude.

It can be postulated that the strengthening external stimulus leads to a convergence in simulated and observed CoM motion, i.e. the stronger the equivalent inertia force experienced by the subject, the more like a passive IP they behave during the single stance phase. It is at least reasonable to suggest that the passive nature of the IP model is sufficient to describe the subject's CoM oscillation in the presence of lateral base motion. That is to say the addition of a further active control element on CoM does not appear to be warranted. While active balance control certainly plays a role in maintaining stability during locomotion on a laterally oscillating deck, the CoM behaviour is adequately described by the dynamics of a passive IP.

It is worth commenting on the IP performance in the case when the lateral forcing and deck oscillation frequencies coincide. This case is shown in Fig. 14, in which the oscillation amplitude and frequency are $20 \mathrm{~mm}$ and $0.9 \mathrm{~Hz}$ respectively. Exceptionally good agreement is observed between the subject and IP behaviour, quantified by the correlation and regression data, $\mathrm{CC} / \mathrm{m}_{\text {reg }}=0.89 / 0.78$ (pos), $0.97 / 0.93$ (vel) and $0.88 / 0.89$ (acc).

As mentioned in relation to Fig. 4, there is a constant phase relationship between the lateral forcing and deck oscillation frequencies. As a result the equivalent inertia force imposed on the subject and IP, at the time of support placement, remains constant from gait cycle to gait cycle and there is no modulation of stride width. In this particular case, the CoP position is well predicted by the balance law, Eq. (6), and as a result the CoM position, velocity and acceleration are also well predicted. Not withstanding the inaccuracy 

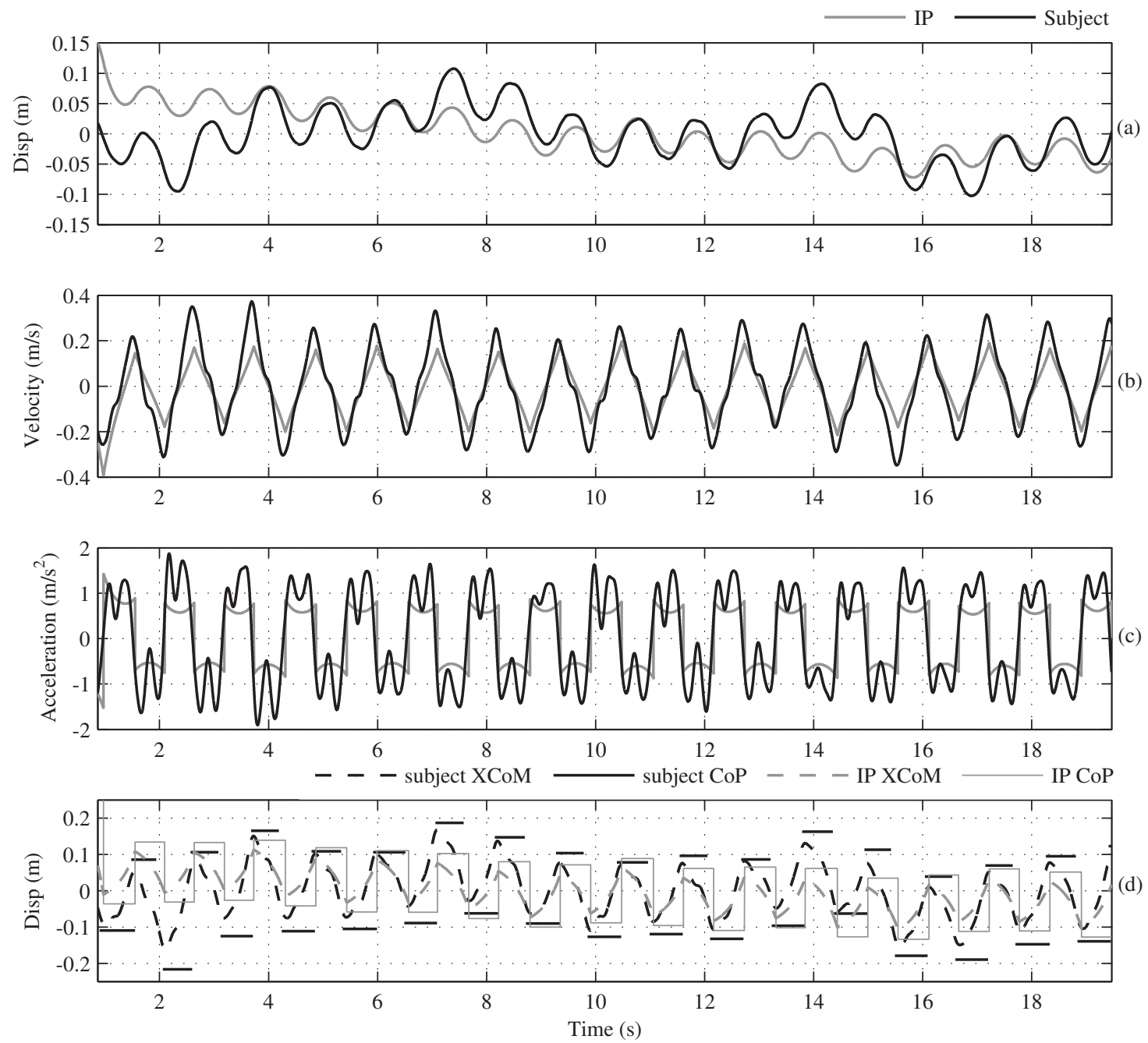

Figure 12: Comparison between subject and equivalent IP, deck oscillation $5 \mathrm{~mm}$ amplitude at $0.3 \mathrm{~Hz}$. (a) CoM position $\left(\mathrm{CC} / \mathrm{m}_{\text {reg }}=0.49 / 0.41\right),(\mathrm{b})$ CoM velocity $\left(\mathrm{CC} / \mathrm{m}_{\text {reg }}=0.95 / 0.57\right),(\mathrm{c}) \mathrm{CoM}$ acceleration $\left(\mathrm{CC} / \mathrm{m}_{r e g}=0.86 / 0.54\right)$ and $(\mathrm{d})$ $\mathrm{XCoM}$ and $\mathrm{CoP}$ position. A constant medial offset of $35 \mathrm{~mm}$ has been applied to the recorded foot position to approximate the average position of the subject's CoP [20].

of support placement position, exemplified in Figs. 12 and 13, Fig. 14 further confirms the suitability of a passive model for CoM oscillatory behaviour.

A limitation of the CoP placement law becomes apparent as the magnitude of deck velocity experienced by the IP grows. According to Eq. (6), the eccentricity of the CoP contains a term proportional to CoM velocity, $\frac{\dot{y}_{0}}{\omega}$, and the additional stability margin, $b_{\min }$.

In the event that the IP and deck have global velocities in the same direction but the deck velocity magnitude is greater than the absolute velocity of the IP, the velocity of the IP relative to the deck will be in the medial direction. As a result, the term $\frac{\dot{y}_{0}}{\omega}$ will be opposite in sign to $b_{\min }$. As deck velocity magnitude increases, $b_{\min }$ is effectively eroded by $\frac{\dot{y}_{0}}{\omega}$ and a 'crossover' step occurs. A crossover step describes the situation in which the IP CoP is placed on the 'wrong' side of the CoM for the imminent step. Figure 15 shows the IP/subject comparison for deck oscillation amplitude and frequency, $35 \mathrm{~mm}$ at $0.6 \mathrm{~Hz}$. The occurrence of crossover steps can be seen in plot (d). This behaviour, although ensuring IP stability, signals the breakdown of the balance law as the IP behaviour no longer resembles human locomotion. For this 

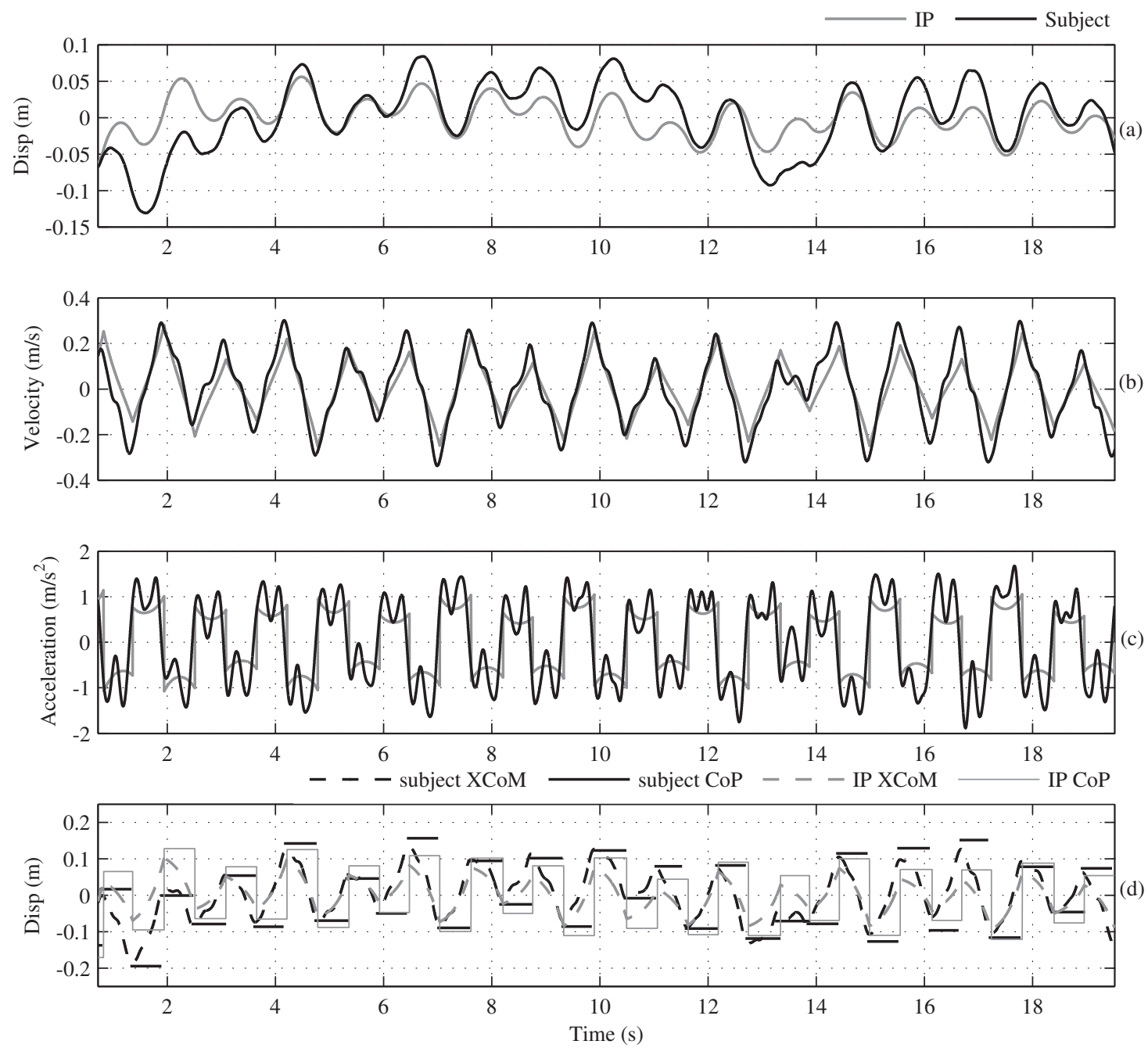

Figure 13: Comparison between subject and equivalent IP, deck oscillation $10 \mathrm{~mm}$ amplitude at $0.5 \mathrm{~Hz}$. (a) CoM position $\left(\mathrm{CC} / \mathrm{m}_{\text {reg }}=0.64 / 0.34\right)$, (b) CoM velocity $\left(\mathrm{CC} / \mathrm{m}_{\text {reg }}=0.93 / 0.66\right)$, (c) CoM acceleration $\left(\mathrm{CC} / \mathrm{m}_{r e g}=0.81 / 0.59\right)$ and $(\mathrm{d})$ $\mathrm{XCoM}$ and $\mathrm{CoP}$ position. A constant medial offset of $35 \mathrm{~mm}$ has been applied to the recorded foot position to approximate the average position of the subject's CoP [20].

reason, comparisons have not been presented for $35 \mathrm{~mm}$ and $50 \mathrm{~mm}$ amplitude tests.

\subsection{Pendulum versus subject CoM motion: population data}

Before the subject/IP comparison can be further investigated, the issue of velocity reference frame must be briefly addressed. As mentioned above, there is a level of uncertainty regarding which reference frame is most appropriate for each comparison test. For example, when focusing on a fixed reference point in the laboratory, the subject may have perceived their CoM velocity in the global reference frame. However when looking down at their feet moving relative to the treadmill deck (or the horizontal stabilising bar), they may have perceived velocity in the local reference frame. This leads to difficulty when trying to generalise IP/subject comparison data for the full population. Therefore the approach adopted here will be to present analyses based on local and global reference frames, in parallel. It can then be assumed that the subject's actual RF during testing lay on or between these two boundaries, an imperfect but practical approach.

Comparisons between each subject and their equivalent IP have been carried out for all 10 subjects. 

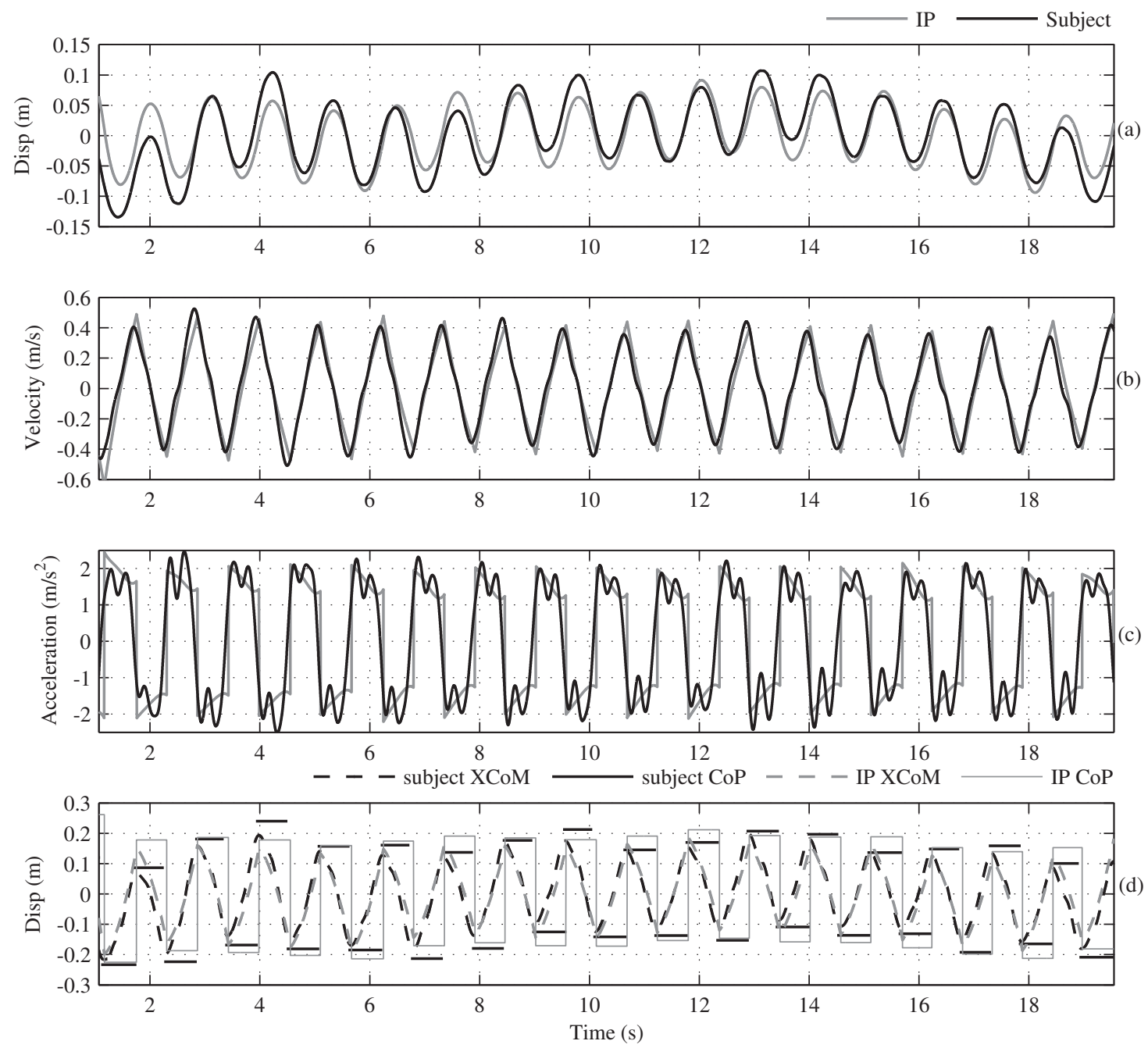

Figure 14: Comparison between subject and equivalent IP, deck oscillation $20 \mathrm{~mm}$ amplitude at $0.9 \mathrm{~Hz}$. (a) CoM position $\left(\mathrm{CC} / \mathrm{m}_{\text {reg }}=0.89 / 0.78\right),(\mathrm{b}) \mathrm{CoM}$ velocity $\left(\mathrm{CC} / \mathrm{m}_{\text {reg }}=0.97 / 0.93\right),(\mathrm{c}) \mathrm{CoM}$ acceleration $\left(\mathrm{CC} / \mathrm{m}_{\text {reg }}=0.88 / 0.89\right)$ and $(\mathrm{d})$ $\mathrm{XCoM}$ and $\mathrm{CoP}$ position A constant medial offset of $35 \mathrm{~mm}$ has been applied to the recorded foot position to approximate the average position of the subject's CoP [20].

All comparison data, for all subjects has been combined for $5 \mathrm{~mm}, 10 \mathrm{~mm}$ and $20 \mathrm{~mm}$ amplitude tests and simulated versus observed CoM motion plotted. The correlation and regression data for both local and global velocity simulations is summarised in table 3 . This values of $\mathrm{CC}$ and $\mathrm{m}_{\text {reg }}$ obtained for the full population confirm the generality of the previous discussion in relation to figures 12, 13 and 14 . CoM velocity and acceleration are predicted more accurately than position in terms of both linearity and magnitude. This is largely due to the fact that the velocity and acceleration data is zero-centred and does not contain the drifting visible in the position data. With the exception of CoM velocity and acceleration during $10 \mathrm{~mm}$ amplitude deck oscillations (based on local velocity simulations), the IP underestimates CoM motion magnitude in all cases. When averaged over the test population, there appears to be negligible difference between local and global simulation predictions. This is not surprising as any variation in CoM motion induced by a change of perception reference frame is likely to be subtle and may change several times during the course of a single test observation. Nevertheless, table 3 confirms the suitability of a passive IP model for CoM motion in the frontal plane. 

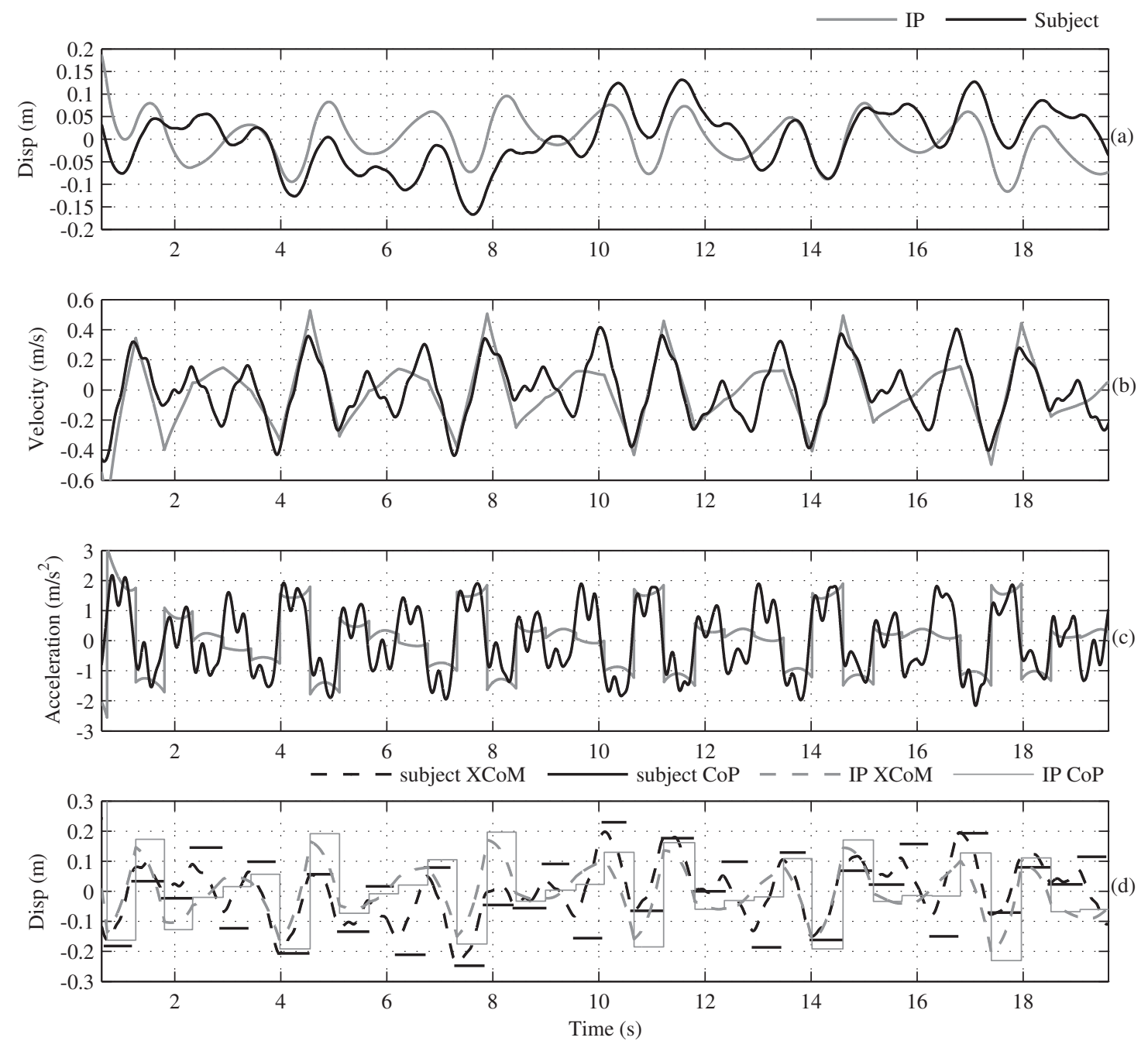

Figure 15: Comparison between subject and equivalent IP, deck oscillation $35 \mathrm{~mm}$ amplitude at $0.6 \mathrm{~Hz}$ demonstrating the occurrence of crossover steps. (a) CoM position, (b) CoM velocity, (c) CoM acceleration and (d) XCoM and CoP position A constant medial offset of $35 \mathrm{~mm}$ has been applied to the recorded foot position to approximate the average position of the subject's CoP, [20].

Table 3: Comparison between subject and equivalent IP CoM motion. Correlation $(\mathrm{CC})$ and regression $\left(\mathrm{m}_{\text {reg }}\right)$ parameters have been determined for the full test population. Comparisons derived from IP simulations based on local and global IP CoM velocity are indicated separately [20].

\begin{tabular}{|c|c|c|c|c|c|c|c|}
\hline & \multicolumn{2}{|c|}{$5 \mathrm{~mm}$} & \multicolumn{2}{|c|}{$10 \mathrm{~mm}$} & \multicolumn{2}{|c|}{$20 \mathrm{~mm}$} \\
\hline & & $\mathrm{CC}$ & $\mathrm{m}_{\text {reg }}$ & $\mathrm{CC}$ & $\mathrm{m}_{r e g}$ & $\mathrm{CC}$ & $\mathrm{m}_{\text {reg }}$ \\
\hline \multirow{2}{*}{ CoM position } & Local & 0.48 & 0.36 & 0.45 & 0.56 & 0.58 & 0.68 \\
\hline & Global & 0.47 & 0.34 & 0.40 & 0.46 & 0.52 & 0.43 \\
\hline \multirow{2}{*}{ CoM velocity } & Local & 0.94 & 0.86 & 0.93 & 1.09 & 0.88 & 0.91 \\
\hline & Global & 0.95 & 0.86 & 0.92 & 0.92 & 0.87 & 0.69 \\
\hline \multirow{2}{*}{ CoM acceleration } & Local & 0.87 & 0.83 & 0.87 & 1.04 & 0.84 & 0.89 \\
\hline & Global & 0.88 & 0.83 & 0.86 & 0.91 & 0.85 & 0.72 \\
\hline
\end{tabular}




\section{Conclusions}

In this paper, an analysis of subject balance behaviour while walking on both a stationary and laterally oscillating treadmill is presented. The aim of this work has been (i) to obtain a better understanding of the root cause or source of the SE GRF, identified in Fig. 3 (d) and (ii) to assess the suitability of the passive IP model for the specific case of walking on a laterally oscillating deck. The approach taken in this investigation was to investigate the behaviour of a relatively small number of subjects, but in greater detail than has typically been the case in the literature thus far.

It has been confirmed that the interaction forces arise directly as a result of the foot placement position, which itself is a function of pedestrian stability. The frequency of stride width modulation was confirmed as the modulus of the difference between the lateral forcing and deck oscillation frequencies. The underlying reason for this was revealed by examining the subject's CoM oscillation and recognising the influence of a sinusoidally varying inertia force induced by deck motion. Furthermore, the degree to which the subject alters their gait is determined by the degree to which their frontal plane stability is impaired by deck motion. Thus the link between frontal plane balance behaviour and generation of the SE component of the lateral GRF has been established. Although there is a large degree of ISV, the fundamental HSI mechanism identified, namely periodic stride width modulation resulting in amplitude modulation of the GRF, can be considered typical for the full test population.

The additional stimulus provided by deck motion has also been shown to cause an increase in mean stride width when compared with walking on a stationary deck. A linear relationship was found between the normalised stride width and deck velocity amplitude. This balance response was exhibited for even the smallest deck oscillation amplitudes imposed in this study.

The experimental observations and analysis presented herein reveal that the IP model, stabilised on a step-by-step basis, is at least qualitatively sound; that is to say the IP model combined with the CoP placement law proposed in [11], generates self-excited forces through the alteration of CoP placement position; a process analogous to amplitude modulation. Examination of actual subject balance behaviour has revealed the same mechanism at work. The IP stability law does not however account for the increase in mean stride width demonstrated by subjects walking on an oscillating structure.

Furthermore, the single stance oscillatory behaviour of subjects walking on the laterally oscillating deck has been found to be well described by a passive IP model for the range of amplitudes $\leq 20 \mathrm{~mm}$ and frequencies $\leq 1.1 \mathrm{~Hz}$. The magnitude of lateral acceleration experienced on full scale structures, including that experienced at the onset of lateral instability, will almost certainly be within this range. Thus the working hypothesis which suggests the need for active balance control during the single stance phase has been disproven. While active balance control based on sensory feedback is certainly utilised in stabilising locomotion on an oscillating structure, its influence on single stance CoM oscillation can be neglected in favour of the passive IP.

In summary, when one considers the need for simulation accuracy and efficiency, on balance, the SDoF passive IP, stabilised by CoP placement, performs remarkably well. More accuracy in CoP placement may be achieved by implementing additional active control elements. However, the potential benefit of this improved accuracy must be balanced against the increased complexity introduced. For the purposes of simulating lateral HSI, the IP/control law system in its current form is judged appropriate and sufficient for implementation as a biomechanical model of frontal plane CoM motion.

\section{References}

[1] P. Dziuba, G. Grillaud, O. Flamand, S. Sanquier, Y. Tétard, La Passerelle Solférino comportement dynamique (dynamic behaviour of the Solférino Bridge), Bulletin Ouvrages Métalliques 1 (2001) 34-57.

[2] S. Nakamura, Field measurements of lateral vibration on a pedestrian suspension bridge, The Structural Engineer 81 (2003) 22-6.

[3] A. Ronnquist, Pedestrian induced lateral vibrations of slender footbridges, Ph.D. thesis, Norwegian University of Science and Technology (2005).

[4] J. M. W. Brownjohn, P. Fok, M. Roche, P. Omenzetter, Long span steel pedestrian bridge at Singapore Changi Airport part 2: Crowd loading tests and vibration mitigation measures, The Structural Engineer 82 (2004) 28-34.

[5] J. H. G. Macdonald, Pedestrian-induced vibrations of the Clifton Suspension Bridge, uk, Proceedings of the ICE - Bridge Engineering 161 (2008) 69-77.

[6] P. Dallard, A. J. Fitzpatrick, A. Flint, S. Le Bourva, A. Low, R. M. Ridsdill Smith, M. Willford, The London Millennium Footbridge, The Structural Engineer 79 (2001) 17-33. 
[7] F. Ricciardelli, A. D. Pizzimenti, Lateral walking-induced forces on footbridges, Journal of Bridge Engineering 6 (2007) 677-688.

[8] E. T. Ingolfsson, C. T. Georgakis, F. Ricciardelli, J. Jonsson, Experimental identification of pedestrian-induced lateral forces on footbridges, Journal of Sound and Vibration 330 (2011) 1265-1284.

[9] S. P. Carroll, J. S. Owen, M. F. M. Hussein, Reproduction of lateral ground reaction forces from visual marker data and analysis of balance response while walking on a laterally oscillating deck, Engineering Structures 49 (2013) $1034-47$.

[10] J. H. G. Macdonald, Lateral excitation of bridges by balancing pedestrians, Proceedings of the Royal Society aMathematical Physical and Engineering Sciences 465 (2009) 1055-1073.

[11] A. L. Hof, M. G. J. Gazendam, W. E. Sinke, The condition for dynamic stability, Journal of Biomechanics 38 (2005) 1-8.

[12] A. L. Hof, R. M. van Bockel, T. Schoppen, K. Postema, Control of lateral balance in walking: Experimental findings in normal subjects and above-knee amputees, Gait \& Posture 25 (2007) 250-258.

[13] A. L. Hof, S. Vermerris, W. Gjaltema, Balance response to lateral perturbations in human treadmill walking, The Journal of Experimental Biology 213 (2010) 2655-2664.

[14] S. P. Carroll, J. S. Owen, M. F. M. Hussein, Crowd-bridge interaction by combining biomechanical and discrete element models, in: Proceedings of the 8th international conference on structural dynamics, Leuven, 2011.

[15] M. Bocian, J. H. G. Macdonald, J. F. Burn, Biomechanically inspired modelling of pedestrian-induced forces on laterally oscillating structures, Journal of Sound and Vibration 331 (2012) 3914-3929.

[16] S. P. Carroll, J. S. Owen, M. F. M. Hussein, A coupled biomechanical/discrete element crowd model of crowd-bridge dynamic interaction \& application to the Clifton Suspension Bridge, Engineering Structures 49 (2013) 58-75.

[17] Charnwood Dynamics Ltd., Leicestershire, UK, Coda mpx30 User Guide, Charnwood Dynamics Ltd, Leicestershire, UK.

[18] V. M. Zatsiorsky, V. N. Seluyanov, L. G. Chugunova, Contemporary Problems of Biomechanics, CRC Press, Massachusetts, 1990, Ch. Methods of determining mass-inertial characteristics of human-body segments, pp. $272-291$.

[19] P. de Leva, Adjustment to zatsiorsky-seluyanov's segment inertia parameters, Journal of Biomechanics 29 (1996) 12231230.

[20] S. P. Carroll, Crowd-induced lateral bridge vibration, Ph.D. thesis, Faculty of Engineering, University of Nottingham (2013).

[21] C. Barker, Some observations on the nature of the mechanism that drives the self-excited lateral response of footbridges., in: Proceedings of the 1st International conference; design and dynamic behaviour of footbridges, Paris, 2002.

[22] F. A. McRobie, Long-term solutions of Macdonald's model for pedestrian-induced lateral forces, Journal of Sound and Vibration 332 (2013) 2846-2855.

[23] M. Townsend, Biped gait stabilization via foot placement, Journal of Biomechanics 18 (1985) 21-38. 